\title{
Reinforced statistical learning of auditory categories
}

Running Head: Reinforced Statistical Learning

\author{
Bob McMurray \\ and \\ Samantha L. Chiu \\ Dept. of Psychological and Brain Sciences \\ University of Iowa
}

\section{Corresponding Author}

Bob McMurray

278 PBSB

Dept. of Psychological and Brain Sciences

University of Iowa

Iowa City, IA 52245

Bob-mcmurray@uiowa.edu

319-335-2408 


\begin{abstract}
A critical step in language acquisition is learning phoneme categories. While L1 learning has been thought to use unsupervised learning (using the distributional statistics of cues), recent research raises the possibility of supervised learning (using teaching signals). Similarly, L2 learning is studied with supervised learning, but unsupervised may also contribute. We develop the reinforced statistical learning paradigm to examine their interaction. Participants first underwent unsupervised learning, hearing a series of non-linguistic sounds whose statistical structure reflected two categories. In subsequent supervised learning, categories either matched or mismatched. Supervised learning was faster when phases matched, though benefits were limited to specific category configurations. Unsupervised learning did not affect the steepness of categorization along the relevant dimension, but it helped subjects learn to ignore irrelevant dimensions. Unsupervised learning may set the stage for supervised learning, but its role may be to determine which dimensions are important, and not to directly acquire categories.
\end{abstract}

Keywords: Auditory Category Learning, Integral Cues, Unsupervised learning, Distributional Learning, Supervised Learning, COVIS 


\section{Introduction}

The ability to categorize sounds is fundamental for spoken language. While phonemes like /b/ or /p/ subjectively feel like stable, invariant percepts, their acoustic instantiation varies across talkers, speaking rates and coarticulatory contexts (Hillenbrand, Getty, Clark, \& Wheeler, 1995; McMurray \& Jongman, 2011). Listeners must abstract over this variation and generalize to new exemplars (e.g., to new talkers or contexts), a key function of categories.

The acoustics of speech can be described in terms of fundamental acoustic dimensions like formant frequencies, pitch, voice onset time and so forth. Languages carve up the possible acoustic/phonetic space into different numbers of categories with different locations. Spanish, for example, has only five vowels, while English has at least 12. As a result, one of the earliest steps in language acquisition is acquiring the categories of the language. Work on first language (L1) acquisition—with roots as an observational science—-has largely focused on unsupervised or statistical learning process, which requires no explicit feedback. In contrast, work on second language (L2) acquisition, with its roots as an applied science, has focused largely on supervised learning (via feedback), analogous to how second languages are taught.

The broader goal of this project is to explore how unsupervised and supervised learning relate. This manuscript has two equally important parts. First, we conduct a critical review of speech category learning in L1 and L2 acquisition with a focus on which mechanisms may be available to which learners. It is important to understand this ecology of learning, as this is what motivated the original proposal of unsupervised for L1. Our review shows more recent work that suggests a different ecology, and this in turn raises the need to consider a tighter integration between supervised and supervised learning. Thus, we present initial results from a new empirical paradigm, reinforced statistical learning, that offers a way to encapsulate some of 


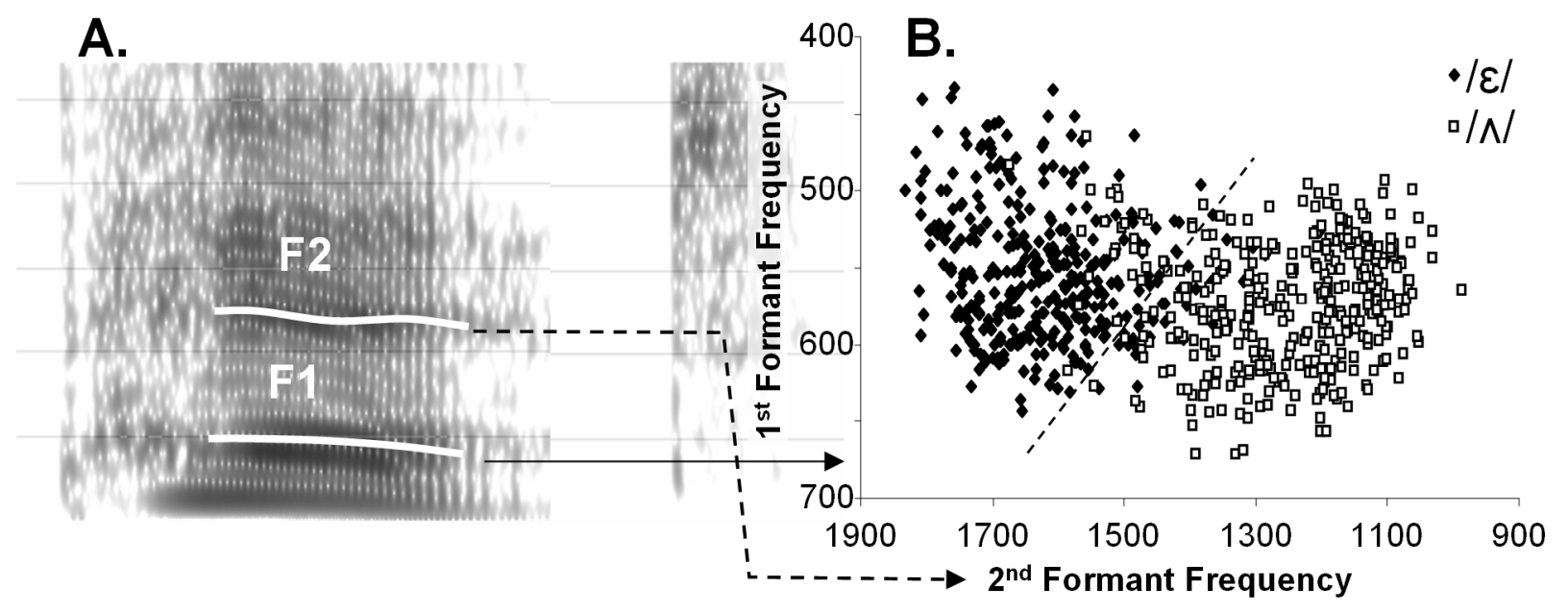

Figure 1: A) Spectrogram of the word "pet". The first and second formants or energy bands (the critical cues for vowel discrimination) are marked. B) Formant frequencies measured for several hundred utterances containing the vowels "eh" and "uh" (Cole, Linebaugh, Munson, \& McMurray, 2010). This shows clustering in a 2D space.

these interactions, and to think about how these kinds of learning mechanisms relate.

\section{Speech Perception as Categorization}

Speech perception is built on categorization (Holt \& Lotto, 2008; McMurray \& Jongman, 2011). Phonemes like "b" or "p" are defined by many variable acoustic cues (Hillenbrand et al., 1995; Jongman, Wayland, \& Wong, 2000), and listeners must generalize across talkers, speaking rates, and contexts to achieve an invariant percept. For example, Figure 1A shows a spectrogram of the word pet. Marked are the first and second formants, whose frequencies are important cues to vowel identity. Typically, the / $/$ / in pet has a slightly lower F1 and a higher $\mathrm{F} 2$ than $/ \Lambda /$ as in putt. However, across many talkers and utterances, there is substantial variability in both (Figure 1B, from Cole et al., 2010). Thus, the categories for $/ \varepsilon /$ and $/ \Lambda /$ can be described as a region of acoustic phonetic space with a multi-dimensional boundary (the dashed line) and some overlap.

These categories are the building blocks of higher-level language (e.g., words). Thus, understanding the learning mechanisms by which they are acquired (and adapted throughout life) is important both for theories of language acquisition and for applied problems like language and 
reading disorders, second language acquisition, and hearing remediation. This has motivated a recent explosion of work examining auditory plasticity, as the auditory system faces a variety of problems, including adapting to distortions (e.g., due to a hearing device) (c.f., Davis, Johnsrude, Hervais-Adelman, Taylor, \& McGettigan, 2005), or to a new accent or dialect (c.f., Bradlow \& Bent, 2008; Maye, Aslin, \& Tanenhaus, 2008). These forms of plasticity are likely mediated by changes in the way that acoustic cues are mapped to categories like phonemes or words. There is also substantial work examining plasticity, specifically in categories. Some of this has been motivated to ask how listeners shift categories (e.g., to adapt to an unfamiliar talker Kraljic, Brennan, \& Samuel, 2008; Norris, McQueen, \& Cutler, 2003); where others have focused on the acquisition of auditory categories.

This work has revealed training paradigms that lead to better learning (Lively, Logan, \& Pisoni, 1993; Logan, Lively, \& Pisoni, 1991; McCandliss, Fiez, Protopapas, Conway, \& McClelland, 2002; Perrachione, Lee, Ha, \& Wong, 2011), predictors of individual differences in learning (Eisner, McGettigan, Faulkner, Rosen, \& Scott, 2010; Perrachione et al., 2011; Wong, Perrachione, \& Parrish, 2007; Wong et al., 2008), and the cortical architecture of learning (Chandrasekaran, Yi, \& Maddox, 2014; Maddox, Chandrasekaran, Smayda, \& Yi, 2013; Yi \& Chandrasekaran, 2016; Yi, Maddox, Mumford, \& Chandrasekaran, 2016).

However, the typical paradigms used in these studies do not capture the richness of the learning mechanisms that are likely deployed in real language acquisition. That is, most work attempts to isolate a single mechanism of learning (e.g., to develop a training procedure for applied goals, or to isolate and demonstrate a specific learning mechanism). But in L1 and L2 acquisition, people may engage multiple forms of learning or use multiple forms of feedback.

Here we focus on two classes of learning mechanisms: unsupervised and supervised. For 
specificity, we define supervised learning as any form of learning in which the learner is given some external signal to group some sounds together and separately from others. This could be explicit in the form of response feedback (e.g., in a training paradigm), but it also could be implicit (e.g., noticing that pet and putt mean different things). Unsupervised learning takes place only through the bottom up analysis of the input, without any explicit feedback or external cues to establish contrast. It occurs during passive exposure (though it may benefit from attention or occur simultaneously with supervised learning).

\section{Unsupervised Statistical Learning in L1 Category Learning}

Classic work suggests infants rapidly tune into the categories of their language during the first year (e.g., Eimas, Siqueland, Jusczyk, \& Vigorito, 1971; Werker \& Tees, 1984) (Galle \& McMurray, 2014; Kuhl, 2004; Tsuji \& Cristia, 2014; Werker \& Curtin, 2005). Prior to 6 months, infants discriminate most speech sounds. These abilities become tuned to the native language by 12-18 months, with decreases in discrimination for non-native contrasts, and increases for contrasts used in the language (Galle \& McMurray, 2014; Kuhl, Stevens, Deguchi, Kiritani, \& Iverson, 2006; Tsuji \& Cristia, 2014). This is not the only pattern: some contrasts are initially hard to discriminate and acquired with experience (Eilers, Wilson, \& Moore, 1977; Narayan, Werker, \& Beddor, 2010). But even these cases supported the idea that this happens early.

Critically at these ages, infants have little access to supervisory signals. While children at any age are unlikely to get explicit feedback on their perception, there may be implicit supervisory signals. For example, lexical knowledge that could alert a learner to the fact that two sounds fall in distinct categories if they contrast distinct meanings (Feldman, Griffiths, Goldwater, \& Morgan, 2013; Metsala \& Walley, 1998); or that a category should be expanded to 
accommodate a deviant input (Norris et al., 2003). Yet infants know few words to support these inferences $^{1}$. Similarly, infants could produce speech and compare their percepts to what they thought they produced, but production is poorly organized at this age. They have also not been taught alphabetic spelling systems which could provide overt labels for auditory categories (Dich \& Cohn, 2013). Thus, most accounts assume that this early acquisition of speech categories proceeds without feedback as a form of perceptual learning (Jusczyk, 1993).

The most prominent of these accounts take the form of unsupervised distributional (statistical) learning (de Boer \& Kuhl, 2003; Maye \& Gerken, 2000; Maye, Werker, \& Gerken, 2003; McMurray, Aslin, \& Toscano, 2009). The idea is that infants track how often specific cue values occur (e.g., how is a first formant frequency of $400 \mathrm{hz}$ heard; Figure 1B). These distributions are shaped by the categories of the language such that each category corresponds to a "cluster". By identifying these clusters, infants can derive the likely categories of the input.

A number of studies have attempted to isolate this mechanism in laboratory studies of learning (Escudero, Benders, \& Wanrooij, 2011; Escudero \& Williams, 2014; Goudbeek, Cutler, \& Smits, 2008; Goudbeek, Swingley, \& Smits, 2009; Maye \& Gerken, 2000; Maye, Weiss, \& Aslin, 2008; Maye et al., 2003). In these studies, learners start by passively hearing a series of stimuli whose cues form statistical clusters. Assessing the results of learning after the fact however is difficult, as listeners do not have any labels or responses that are associated with the stimuli (that would have turned it into a supervised learning task). As a result, researchers typically infer category learning by examining the pattern of discrimination among pairs of stimuli with the inference that discrimination should be reduced for tokens within the same

\footnotetext{
${ }^{1}$ That's not to say that infants cannot engage in supervised learning for auditory categories - a number of laboratory studies suggest they can bootstrap off of lexical contrasts to refine auditory discrimination (Thiessen, 2007; Yeung \& Werker, 2009). Rather in young infants, there may not be sufficient words known to support this as the general mechanism of development.
} 
category and heightened for tokens that span two categories. While this mechanism was first proposed as a learning mechanism during infancy, it has been empirically demonstrated in adults as well and as we describe it is likely relevant for L2 acquisition (Escudero et al., 2011; Escudero \& Williams, 2014; Goudbeek et al., 2008; Goudbeek et al., 2009).

Distributional learning can only be indirectly observed via patterns of discrimination. Consequently, while it is clear that passive exposure drives some sort of change in the system, it is not clear what the locus of this change is. The standard assumption is that unsupervised learning leads to new categories. Most computational models thus put the locus of learning at the mapping between regions of the cue-space and category representations (de Boer \& Kuhl, 2003; McMurray et al., 2009; Toscano \& McMurray, 2010; Vallabha, McClelland, Pons, Werker, \& Amano, 2007). However, others propose that unsupervised learning alters the representation of the acoustic space (below the level of categories) to emphasize regions corresponding to category prototypes (Gauthier, Shi, \& Xu, 2007; Guenther \& Gjaja, 1996).

Thus, even as there is a clear consensus for some form of unsupervised learning and empirical evidence for such learning, the specific computational loci of learning are still unclear.

Limits of unsupervised learning in L1 acquisition. In recent years, converging lines of evidence have started to challenge this consensus. First, analysis of the statistical distributions of infant directed speech suggests cases in which categories are not reflected by clusters in the input (Bion, Miyazawa, Kikuchi, \& Mazuka, 2013), and in fact, it now appears that infant directed speech makes categories less discriminable relative to adult-directed speech (Cristia \& Seidl, 2013; Martin et al., 2015; McMurray, Kovack-Lesh, Goodwin, \& McEchron, 2013). This suggests that unsupervised mechanisms may not fully account for perceptual development.

Second, work in L1 acquisition has shown speech category acquisition to be a protracted 
process. During late infancy, infants go through a phase of determining which cues are relevant for contrasting words, learning that cues to talker are not relevant, but cues to voicing or vowel contrasts are (Dietrich, Swingley, \& Werker, 2007; Hay, Graf Estes, Wang, \& Saffran, 2015; Rost \& McMurray, 2009, 2010). This last through 24 months.

More impressively, many studies have now tested children on the kinds of fine-grained identification tasks commonly used with adults (Hazan \& Barrett, 2000; McMurray, Danelz, Rigler, \& Seedorff, 2018; Nittrouer, 2004; Nittrouer \& Miller, 1997; Slawinski \& Fitzgerald, 1998). In these tasks, children hear tokens from a continuum spanning two categories (e.g., /b/ to /p/) and explicitly label them, enabling researchers to quantify the precise shape of the category. These studies uniformly show that categories continue to sharpen throughout childhood and sometimes through adolescence. This is a time during which there may be ample access to implicit supervised learning signals like lexical knowledge, spelling and production.

Putting this work together, we must consider the possibility that the infant results have been misinterpreted. Prelinguistic infants may not "acquire" the categories of their language via unsupervised statistical learning. Rather unsupervised learning may serve to structure a perceptual map (along the lines of Gauthier et al., 2007; Guenther \& Gjaja, 1996), and this structure can bias discrimination as measured by the coarse tools we use with infants. However, this is not the same as learning categories, which may be imposed upon this representational space later, by implicitly supervised learning (via the lexicon, reading/spelling instruction, speech production or other mechanisms). At this point, this hypothesis remains speculative because no laboratory studies have examined both supervised and unsupervised learning together, and we do not have measures of the pre-categorical auditory map. Part of the goal of the present project is to present a preliminary paradigm to help address this. 


\section{Supervised Learning in $\mathbf{L} 2$ acquisition.}

Most work on adult L2 learning has emphasized supervised learning in which learners receive feedback during training. Whereas work on $\mathrm{L} 1$ learning has been profoundly motivated by a detailed consideration of the ecology of learning (e.g., what learning mechanisms might be available given the capacities of the learner and the environment), L2 work has focused on more basic questions about supervised learning. This is appropriate for two reasons. First, L2 speech perception skills are exceedingly difficult to acquire, and many adults never learn to discriminate non-native sounds at all (Strange \& Shafer, 2008). As a result, remediating these difficulties may require focused training (e.g., in a language lab). Given that supervised learning is widely seen as more efficient, this may be a better place to start. Second, in the ecology of L2 learning, learners likely have access to implicit supervisory signals: they can produce speech, they are typically taught to spell in the $\mathrm{L} 2$, and there is a teacher available.

Supervisory signals typically come in three forms. First, sounds may be explicitly labeled (e.g., "this is an A") as in a study/recall paradigm (Chandrasekaran, Yi, et al., 2014; Yi \& Chandrasekaran, 2016). Second, there may be direct feedback on performance. In these paradigms, modeled on operant learning, learners hear a stimulus, choose a category and receive feedback (Chandrasekaran, Yi, et al., 2014; Earle \& Myers, 2015; Lively et al., 1993; Logan et al., 1991; Maddox et al., 2013; Mirman, Holt, \& McClelland, 2004; Perrachione et al., 2011; Reetzke, Maddox, \& Chandrasekaran, 2016; Smits, Sereno, \& Jongman, 2006; Yi \& Chandrasekaran, 2016). Here, there is a clear error signal, which can correct any incorrect mappings. Finally, the supervisory signal may be an implicit signal that indicates the category to which a token belongs. For example, in a series of implicit supervised learning paradigms by 
Holt and colleagues, learners play a game in which they associate categories of sounds with (for example) the location of an alien attack (Lim \& Holt, 2011; Liu \& Holt, 2011; Roark \& Holt, 2018; Wade \& Holt, 2005). The type of supervisory signal matters: response-based feedback is more robust than explicit labeling (Chandrasekaran, Yi, et al., 2014; Yi \& Chandrasekaran, 2016), and operant training is more robust than an implicit signal (Roark \& Holt, 2018).

This supervised paradigm has led to several important findings. For example, some of the difficulty in learning new L2 speech categories may derive from the similarity of the new categories to the L1. When the to-be-learned categories are dissimilar from existing contrasts in the language, sounds may be more learnable (Earle \& Myers, 2015; Pisoni, Aslin, Perey, \& Hennessy, 1982). Moreover, learning may benefit from variability in irrelevant factors (e.g., in the talker voice) (Lively et al., 1993; Logan et al., 1991), though the optimal structure of the training set may differ across individuals (Perrachione et al., 2011).

A great deal of recent work has been motivated by the COVIS model of visual categorization (Ashby \& Alfonso-Reese, 1998; Ashby \& Maddox, 2011). COVIS posits two learning systems: one for categories defined by a boundary along a single cue (rule-based or RB categories), and a second for categories that require integrating cues along a multidimensional (diagonal) boundary (information integration or II categories). II categories are more difficult to acquire than RB (Ashby \& Maddox, 2011). This may be due to learners attempting to misapply (or perseverate on) an erroneous single dimensional rule (Freedberg, Glass, Filoteo, Hazeltine, \& Maddox, 2017). II learning is also more sensitive to precisely timed feedback (Maddox, Ashby, \& Bohil, 2003; Maddox \& Ing, 2005), and unsupervised learning may only be only possible for RB configurations (Ashby, Maddox, \& Bohil, 2002b; Ashby, Queller, \& Berrety, 1999).

The RB advantage has been shown in auditory categories (Chandrasekaran, Yi, et al., 
2014; Roark \& Holt, 2018). However, it is widely acknowledged that the kinds of categories used in human language are likely II, as most are highly multi-dimensional and context sensitive (Chandrasekaran, Koslov, \& Maddox, 2014; Chandrasekaran, Yi, et al., 2014). Moreover, COVIS is controversial and many of the effects supporting it have other explanations (Lewandowsky, Yang, Newell, \& Kalish, 2012) (Richler \& Palmeri, 2014, for a review). Minimally, however, COVIS raises the importance of examining different category structures.

Unsupervised in L2. While work on L2 category learning has been dominated by supervised learning, unsupervised learning is not irrelevant. In a classroom context, the bulk of a learner's time is not spent learning to discriminate speech sounds. Thus, there is substantial opportunity for unsupervised learning. Moreover, unsupervised learning is likely relevant to immersive learning experiences (study abroad, migration, etc).

There are a number of key studies. First, McCandliss et al. (2002) used a supervised learning paradigm to teach Japanese speakers the /l/ vs. /r/ contrast. Critically the statistics of the cue values (across training trials) were manipulated similarly to an unsupervised distributional learning experiment (with clusters corresponding to categories). This improved learning, suggesting that even during supervised learning, unsupervised statistical learning is ongoing (see also Giguère \& Love, 2013; Roark \& Holt, 2018). Second, recent studies have demonstrated unsupervised learning in adults, with real speech sounds (Escudero et al., 2011; Escudero \& Williams, 2014; Goudbeek et al., 2008; Maye \& Gerken, 2000), and complex non-speech sounds (Goudbeek et al., 2009). This suggests unsupervised learning is operative in adult L2 learners.

\section{Interactions of supervised and unsupervised learning}

The foregoing review suggests neither unsupervised or supervised learning alone captures 
either L1 or L2 category learning. This is due to ecological factors (L2 learners are learning from exposure as well as from any focused training), and computational limits (unsupervised learning appears insufficient to capture some L2 phenomenon). Rather both L1 and L2 learning likely to require a mix which may vary across domains or learners. For example, given the limitations of infancy, L1 learning may start as an unsupervised process, but give way to implicit supervised learning, as the lexicon, speech production, and reading skills develop. In contrast, L2 learning may start out with focused classroom training followed by periods unsupervised learning (immersive experiences). Thus, it is important to ask how these mechanisms jointly contribute to learning and how they work under various sequences or schedules.

Only a small body of work directly addresses the interaction of mechanisms, and none consider the issue of sequencing. Several studies by Goudbeek and colleagues have directly compared supervised and unsupervised learning in the same stimuli. They generally report that supervised learning is more robust than unsupervised (Goudbeek et al., 2008; Goudbeek et al., 2009). This raises the possibility that supervised learning could block or overwrite prior unsupervised learning; in this case, unsupervised learning may ultimately play little role in the final structure of the categories (even as it serves as a useful way to get off the ground during infancy). However, McCandliss et al. (2002) suggests both may benefit from the same kind of distributional information, suggesting there the utility of considering these concurrently.

COVIS is the only theoretical model that makes strong claims about both (though as we discuss in the conclusions, SUSTAIN, may also offer insight; Love, Medin, \& Gureckis, 2004). COVIS (Chandrasekaran, Koslov, et al., 2014) predicts that unsupervised learning is only possible for one-dimensional RB categories (Ashby, Maddox, \& Bohil, 2002a). Given that human speech sounds are generally thought to be II, this would seem to challenge the utility of 
unsupervised learning for speech at all. However, this claim has not yet been tested in auditory categories. Moreover, many studies of unsupervised learning only manipulate a single dimension (Escudero et al., 2011; Escudero \& Williams, 2014), though others show learning for multi-dimensional categories (Goudbeek et al., 2008; Goudbeek et al., 2009). It is not yet clear if certain classes of auditory categories are differentially susceptible to unsupervised learning.

\section{Summary}

Real-world auditory category learning is not simple. The canonical L1 case was long thought to be largely a matter of unsupervised statistical learning. However, studies of the speech directed to children (Bion et al., 2013; Cristia \& Seidl, 2013; McMurray et al., 2013) suggests this is not sufficient and the locus of unsupervised learning-whether it acquires categories or shapes auditory representations—is not clear. The fact that perceptual development is protracted (Hazan \& Barrett, 2000; McMurray et al., 2018; Slawinski \& Fitzgerald, 1998) raises the possibility of implicit teaching signals from lexical development (Metsala \& Walley, 1998; Walley, Metsala, \& Garlock, 2003) or reading skills (Dich \& Cohn, 2013).

In contrast, L2 learning has been dominated by supervised learning and revealed key principles, like the fact that categories may vary in difficulty according to their dimensional structure (Chandrasekaran, Koslov, et al., 2014; Roark \& Holt, 2018). However, unsupervised learning is possible in adults (Escudero et al., 2011; Escudero \& Williams, 2014; Goudbeek et al., 2008; Goudbeek et al., 2009), and it is relevant to L2. There is very little work documenting the way that unsupervised and supervised learning may relate to each other.

A critical limitation is that we cannot observe learning mechanisms operating in real language learning. Actual language learning is too slow and the environment is too complex, 
with a mixture of supervisory and statistical factors at play simultaneously. As a result, the evidence for the involvement of specific mechanisms is often circumstantial. For example, in L1 acquisition, the case for unsupervised learning is made by 1) analysis of the statistics of real speech sounds; 2) computational models; and 3) laboratory studies that can isolate a form of learning in a highly simplified way to show that it is feasible over a short time scale. These are serious limitations with no obvious solution. However, laboratory studies of category learning are critical to this enterprise by allowing us to isolate and control aspects of learning (the distributions of the exemplars, the availability of feedback, the nature of the boundaries). What is thus needed is a laboratory learning that can simultaneously investigate the joint contributions of supervised and unsupervised learning.

\section{The Present Study}

We propose a new laboratory paradigm that offers a parametrically manipulable approach to studying supervised and unsupervised learning simultaneously. We call this the reinforced statistical learning paradigm and it combines both forms of learning sequentially. The initial implementation that is presented here captures a sequence that is characteristic of L1 acquisition, in which supervised learning builds on prior unsupervised learning (see Castro, Wasserman, \& Lauffer, 2018; Mirman, Magnuson, Estes, \& Dixon, 2008, for analogues in other domains).

The use of natural speech in such investigations creates two challenges. First, natural speech categories are multi-dimensional and difficult to fully control. As a result, many studies of category learning that use real speech isolate a single dimension (e.g., voice onset time, duration), even as the true phonological categories comprise multiple dimensions. This is a reasonable simplification. However, given the claim from COVIS that multi-dimensional (II) 

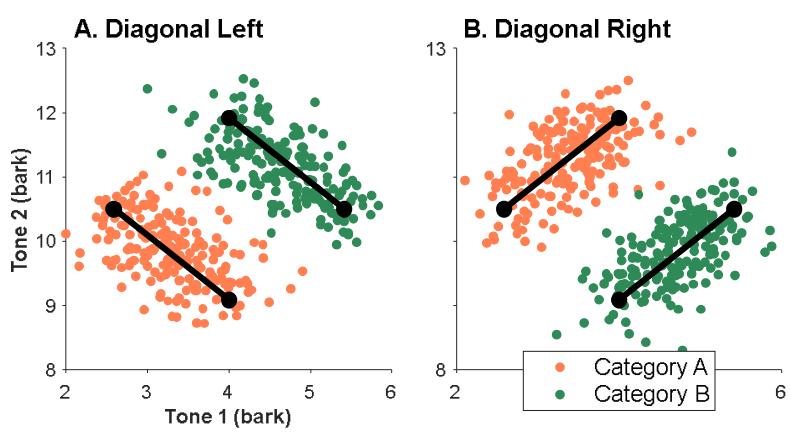

E. Training

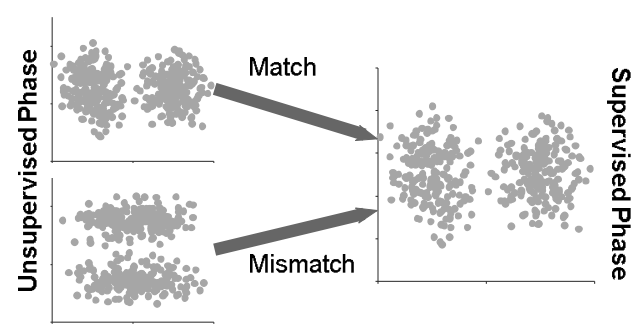

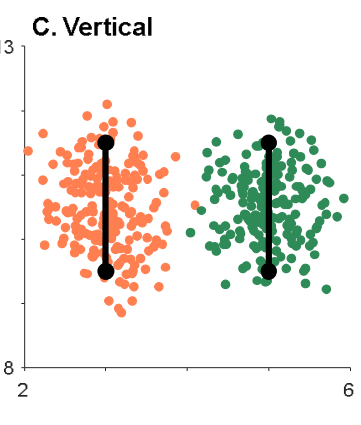
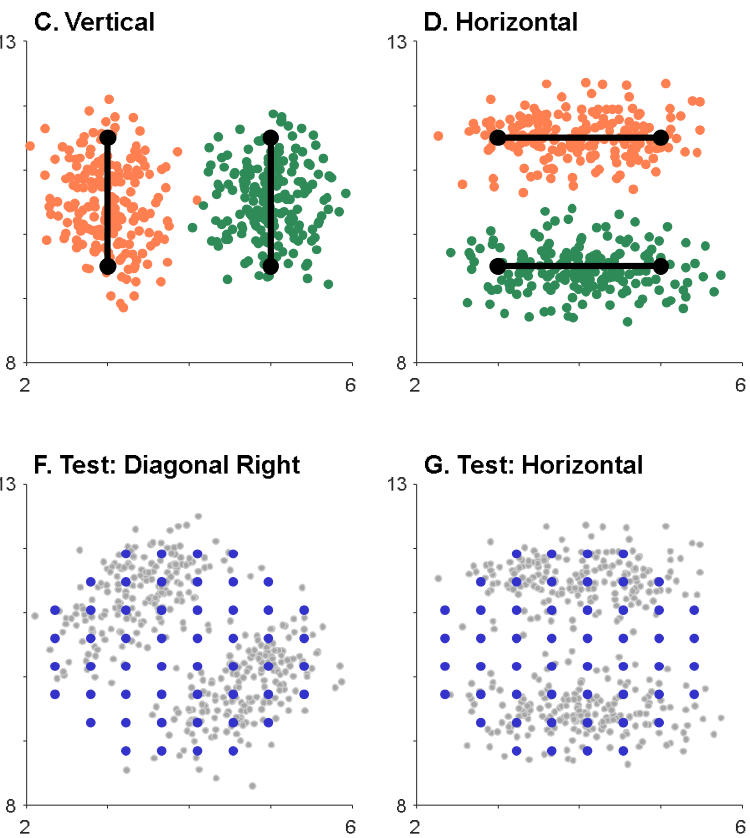

Figure 2: A-D) Representative distributions of stimuli for the four category configurations. Each point is the Tone $1 \times$ Tone 2 frequency of one exemplar. Orange points are designated Category A; Green are Category B. Black points and lines represent the prototype segments for that category. E) Unsupervised distributions (left) can either match or mismatch the supervised distribution (right). F,G) Subjects are tested on a subset of an $8 \times 8$ grid that uniformly the samples the space for diagonal $(\mathrm{F})$ and horizontal/vertical $(\mathrm{G})$ configurations.

categories are learned differently than single dimensional ones (RB), this is a concern. Second, a second challenge in studying auditory category learning in adults is that the learners come to the lab with a large set learned categories (e.g., the 44 phonemes of English). These can interfere with acquiring new ones (Earle \& Myers, 2015; Francis, Ciocca, Ma, \& Fenn, 2008). As a result, laboratory learning studies with speech input may reflect more the process of overcoming interference than the true learning process.

As a result of these challenges, auditory category learning has been extensively investigated with complex non-speech sounds (Goudbeek et al., 2008; Goudbeek et al., 2009; Mirman et al., 2004; Roark \& Holt, 2018; Smits et al., 2006; Wade \& Holt, 2005). These do not undergo interference from existing speech categories and are easy to generate parametrically. Non-speech auditory categories can be learned via similar supervised and unsupervised 
mechanisms (Goudbeek et al., 2008; Goudbeek et al., 2009). When these have been compared to real speech, results generally pattern quite closely (Liu \& Holt, 2011; Mirman et al., 2004).

The present project builds on this tradition using non-speech stimuli. Stimuli are modeled roughly after vowels with two formants. Each stimulus comprises two tones played simultaneously where the frequency of each tone can be used as a feature or a dimension. The frequency of each tone is randomly generated from one of two clusters, corresponding to categories (Figure 2). We examined four types of categories: vertical categories defined solely by the frequency of the lower tone (Tone 1; Figure 2C); horizontal categories defined solely by the upper tone (Tone 2; Figure 2D); diagonal-left categories in which the boundary integrates both tones such that tone frequencies are correlated across the boundary (Figure 2A); and the converse diagonal-right categories (Figure 2B). The former two configurations can be considered RB and the latter as II categories (in the language of COVIS).

Learners start with passive exposure to a series of stimuli whose statistical distribution reflects one of the four configurations. Learners engaged in a non-demanding cover task to keep them attentive but were not told about the categories. After 300 trials, they then completed an operant task to learn to categorize the stimuli. Critically, the boundaries imposed during supervised training can either match or mismatch the unsupervised configuration (Figure 2F). Finally, learners were tested on a grid of many possible Tone $1 \times$ Tone 2 (Figure 2F,G) combinations to precisely characterize the shape of the categories.

We infer unsupervised learning as a difference in supervised performance as a function of whether the boundaries match across phases. If learners acquire something useful from unsupervised learning, we should see a benefit when the boundaries match. In the extreme, if unsupervised learning is sufficient for category learning, learners should start near ceiling in the 
match condition, and quite poor in the mismatch. We further manipulated the configuration of the unsupervised and supervised paradigms (diagonal-left, vertical, etc) in a 4 (supervised configuration $) \times 2$ (unsupervised / unsupervised match) between-subjects design. If unsupervised learning is moderated by the structure of the category, we would expect interactions of the supervised paradigm and unsupervised/supervised match. We quantify performance both in terms of overall accuracy during training (the learning curve) and by a systematic examination of the whole category structure at test. The latter can potentially reveal systematic distortions (or corrections) to the category structure by unsupervised learning.

\section{Experiment}

This experiment was conducted online. In part this was due to the need to run a large number of subjects for what was necessarily a between-subjects design. In part, this was due to limits on in-person testing due to the Covid-19 pandemic. Online work has been led by survey work, and work in domains like vision, memory and decision making. Auditory research has been slow to adopt online testing because it is hard to ensure consistent auditory quality (participants use their own headphones and soundcards) and background noise cannot be controlled.

In recent years, laboratories have adopted techniques (also used here) that ensure that the subject is wearing some type of headphones, and that the soundcard and headphones are of reasonable quality. With these techniques, they show strong matches between laboratory and online versions of the same paradigm (e.g., Cooke, Barker, Lecumberri, \& Wasilewski, 2011; Slote \& Strand, 2016). Thus we adopt a simple test (based on Geller et al., submitted; Woods, Siegel, Traer, \& McDermott, 2017) to ensure reasonably good audio presentation over headphones, and numerous quality checks in the data itself. 


\section{Participants}

Participants $(n=221)$ were monolingual English speakers between ages 18 and 70 with self-reported normal hearing and normal vision. Participants were recruited through Amazon Mechanical Turk and the University of Iowa undergraduate subject pool. Both groups were consented through a University of Iowa approved IRB protocol. Amazon MTurk participants were paid \$11.75 and University of Iowa undergraduates participated for course credit.

Because this was an online study, we were concerned about motivation and engagement (or simply taking a break in the middle). Thus, limited total procedures to 1.75 hours; subjects who took longer were excluded $(n=29)$. An additional participant withdrew early. Finally, 28 people were excluded by quality checks in the experimental tasks (see Procedure). The final sample included 163 participants (mean age $=36.08$ years $\mathrm{SD}=12.26 ; 56 \%$ male; $78 \%$ white).

\section{Design}

Subjects learned a two category structure (category A vs. B) in a two dimensional space that was defined by the frequencies of each tone in the complex. There were four possible configurations (Figure 2A-D). Two configurations, diagonal-left and diagonal-right, were II structures. In the diagonal-left configuration (Figure 2A), Category A was defined by both low Tone 1 and Tone 2 frequencies, while Category B had high frequencies in both. In the diagonalright configuration (Figure 2), Category A was defined by a low Tone 1 but a high Tone 2, while Category B was the reverse. The other two configurations, vertical and horizontal, were RB structures. In the vertical configuration (Figure 2C), Category A had a low Tone 1 and Category B had a high Tone 1; Tone 2 was irrelevant. In the horizontal configuration (Figure 2D), 
Category A had a low Tone 2 and Category B had a high Tone 2, Tone 1 was now irrelevant.

Subjects were first randomly assigned to the configuration that would be taught in the supervised phase. This was treated as the true category structure for that participant. Next, participants were assigned a configuration for the unsupervised phase. This configuration either matched or mismatched the supervised configurations. Mismatching configurations were always within the same class (II or RB) as supervised. For example, a participant in the mismatch condition who learned the vertical (RB) configuration in the supervised phase would be assigned the horizontal configuration for unsupervised learning (Figure2E).

Unsupervised training consisted of a passive listening task of 300 trials. Stimuli from Category A and B were evenly split between trials. Stimuli were randomly sampled from the space to generate clusters (Figure 2A-D) for each category. Subjects were given a cover task during this phase to keep them engaged (press the space bar when you hear a "ringing sound"). There were 12 catch trials (in which the ringing sound was present) interspersed throughout, such that one cover task trial would appear randomly in every block of 25 trials.

Supervised training was a $2 \mathrm{AFC}$ task. On each trial, subjects heard a stimulus, chose the category, and received feedback. There were 12 blocks. Each block consisted of 32 training trials with feedback, followed by eight trials without feedback as a periodic test ${ }^{2}$. Each stimulus in the training set was unique. There were 384 trials with reinforcement and 48 without.

The testing phase task was identical to the periodic test trials (no feedback). Testing used a roughly circular grid with an 8-step diameter in both Tone 1 and Tone 2 space. This was designed to be an evenly spaced distribution that sampled the entire category space similarly for all four configurations (Figure2 F,G; e.g., if we had used a square grid, the corners would be

\footnotetext{
${ }^{2}$ Initial analyses found no difference between these trials and the trials with feedback, so analysis of training collapsed all of the trials together.
} 
relatively novel if the subject were exposed to a diagonal configuration). This comprised 52 unique stimuli. These were repeated four times (in random order) for a total of 208 trials. This grid was the same across all participants.

Stimuli for the supervised and unsupervised phases were randomly generated using the procedure described below. Because of limits of the online testing platform, this could not be done for each subject. Thus, four sets of stimuli were generated for each configuration for both unsupervised and supervised training and participants were randomly assigned to a set. We also counterbalanced the assignment of responses keys to category A or B for each particpant.

\section{Category Structures}

Stimuli were comprised of two tones played simultaneously. We designated the lower frequency tone as Tone 1 and the higher as Tone 2 . The range of possible frequencies for Tone 1 and 2 did not overlap. Categories were defined in Bark space (rather than $\mathrm{Hz}$ ) to ensure roughly equal discriminability per unit of Bark.

Categories are defined by prototypes that describe the central tendency of each category with some variance. In order to ensure that the II categories required a diagonal boundary, we used elongated categories, rather than circular categories. Thus, prototypes were defined by a line segment in Tone $1 \times$ Tone 2 space. These protoype segments were all permutations of the same four values of Tone 1 and Tone 2 to ensure that categories were equally spaced (and hence that categories were similarly discriminable across conditions). For the RB categories, the prototype segments had endpoints which had Tone 1 frequencies of either 3 or 5 bark, and Tone 2 frequencies of 9.5 or 11.5 bark. For the II categories, these points were rotated by $45^{\circ}$ to $<4.0$, $9.09\rangle,\langle 2.59,10.5\rangle,\langle 4.0,11.92\rangle,<5.41,10.5\rangle$. The prototype for each category was the 
segment connecting two of the four category centers (Figure 2 A, D).

A given stimulus was generated randomly in three steps. First, a category (A or B) was chosen. Next, we chose a point along the corresponding prototype segment from a uniform distribution (all locations equally likely). Next the two tones of the stimulus were drawn from a multivariate Gaussian distribution. This distribution had a mean specified by this random point. The variance (in each direction) was set to the distance between segments divided by 5.5 (the categories were separate by $d=5.5$ ). Figure 2A-D shows representative runs of this procedure.

\section{Procedure}

Participants recruited through SONA first completed an online informed consent, then a demographic survey, followed by a headphone screener. Participants recruited through Mechanical Turk first completed the demographic survey, then the headphone screener, followed by the online informed consent. Next participants were randomly assigned to a category configuration for the supervised conditions and a match condition for the unsupervised phase.

Audio screener. Participants completed an audio screener to be eligible to participate. Participants were instructed to wear headphones throughout the experiment. During the screener they were first allowed to adjust their volume to a comfortable level. They then completed six trials. On each trial, they heard a series of three tones, identical in frequency and duration. One had a lower amplitude than the others and participants reported which was the quietest. Stimuli were stereo, and left and right channels were in opposing phases. Thus, the stimulus only be heard with headphones; in free field, the left and right sounds cancel out. Tones were $200 \mathrm{hz}$, a low frequency that is often cut off by low-end sound cards. Participants were included if they reported four of six trials correctly. Participants were allowed to attempt the headphone screener 
up to three times and were excluded if they failed all attempts $(n=86)$.

Unsupervised Training. Participants were instructed to attend to all stimuli and respond with space bar when a ringing sound was heard. An example was given prior to the task. Participants were told to not access other internet or reading materials during the task. Participants heard a continuous stream of sounds with a $200 \mathrm{msec}$ inter-stimuli interval. Participants were excluded immediately after this task if they did not respond to at least $75 \%$ of cover task trials $(n=23)$.

Supervised Training. Next, participants completed the supervised phase. Participants were told they were to learn two sound categories, that each was assigned to either the F or J key and they would receive feedback on some trials. Examples of audio feedback (correct and incorrect sound) were given. Each trial began when the word "Play" appeared on the center of the screen. The participant then pressed the space bar to hear the stimulus. The participant was then prompted to respond with a keypress. On trials with feedback, participants immediately received audio and visual feedback (i.e., a correct sound with a green check or incorrect sound with a red x, respectively). A 60 second break was given between blocks.

We were concerned about motivation and task engagement since participants were not tested in the lab. Thus, participants were excluded on performance during this phase for two reasons. First, they were excluded immediately after this task if they did not score at least $33 \%$ correct (below chance). Second, after the experiment, we scanned for runs of the same response (indicative of guessing). Any participant that responded with the same key on 8 consecutive trials for four or more blocks of trials was excluded $(n=5)$.

Test. All participants completed the same test trials after supervised training. This task was identical to the previous task except feedback was not given. The procedure was the same as 
the supervised training trials. A 60 second break was given between blocks.

\section{Stimuli}

Stimuli consisted of complex sounds whose frequencies were drawn from the distributions specified for each category configuration (Figure 2). Stimuli were generated automatically using the same Matlab script that randomly generated the tone frequencies. Stimuli were $850 \mathrm{msec}$ long, consisting of $750 \mathrm{msec}$ of audio with a $50 \mathrm{msec}$ of silence before and after audio. The envelope started with a $50 \mathrm{msec}$ linear ramp, then a $650 \mathrm{msec}$ steady state, followed by a $50 \mathrm{msec}$ linear ramp down. The amplitude of Tone 1 was $75 \%$ that of Tone 2 (as these sounded slightly more equal). Sampling rate was $44.1 \mathrm{kHz}$.

Cover task. The oddball stimulus for the cover task had an identical length and envelope as the training stimuli. However, category centers of Tone 1 and Tone 2 were outside the range of the regular stimuli. Tone 1 frequencies were either 6 or 8 bark, Tone 2 frequencies were either 12 or 14 bark. To further distinguish the oddball, Tone 1 amplitudes were .75; Tone 2 amplitudes were .9; and amplitudes were modulated from .5 to 1 at $20 \mathrm{~Hz}$ to create a ringing effect.

During unsupervied training, stimuli were presented at a fast rate; consequently on catch trials we would have had to pause stimulus presentation to enable sufficient itme for a response. This would have made them unnaturally easily. To avoid this issue, stimuli for catch trials actually consisted of two stimuli - the ringing tone, followed by a training stimulus (sort of a fake). This second stimulus was presented at the same ISI after the ringing tone. The frequencies should have been drawn from the same categories as the subject was being exposed to. However, due to a scripting error, the second catch stimulus used the same (vertical) category configuration for all subjects (this was only 12 trials out of over 300 so it was a minor issue). 
Test Stimuli. To encompass the entire category space, we started with an $8 \times 8$ grid. Tone 1 ranged from 2.25 to 5.75 bark, and Tone 2 from 8.75 to 12.25 bark. The grid was offset by 0.1 bark in order to avoid sampling a category center. Tones too far from the primary category space were removed from the test grid, resulting in 52 test stimuli. The amplitude envelopes and duration of the test stimuli were identical the tones used in training.

\section{Results}

Overall, participants learned the categories well and rapidly. Over the first block of 40 trials, participants averaged $74.7 \%$ correct $(\mathrm{SD}=12.8 \%)$ and they reached $82.9 \%$ correct $(\mathrm{SD}=11.5 \%)$ by the end of the training.

We conducted our analysis in two parts. First, we examined accuracy on the training trials. Here, the primary question was whether the match between the unsupervised and supervised configurations affected performance. We also asked if some types of categories (e.g., $\mathrm{RB}$ or II) were learned more easily, and whether this moderated the effect of unsupervised/supervised match. Next, we examined the testing trials to determine if those same factors influenced the final shape and structure of the categories.

This was our first study using an online platform for auditory perception or for a training study (which requires greater engagement). We were concerned that some subjects may have failed to learn the categories for lack of attention, a distracting environment, or poor auditory quality. While some exclusion criteria were meant to rule out obvious factors, subjects' performance was the ultimate judge. However, as the experimental factors were predicted to reduce overall performance in lawful ways, it was important to be careful with excluding subjects on the basis of poor performance. 
Table 1: Sample sizes in the complete dataset and in the restricted set as a function of condition.

\begin{tabular}{|c|c|c|c|c|}
\hline \multirow{2}{*}{$\begin{array}{c}\text { Supervised } \\
\text { Configuration }\end{array}$} & \multicolumn{2}{|c|}{$\begin{array}{c}\text { All Subjects } \\
\text { Unsupervised Configuration }\end{array}$} & \multicolumn{2}{|c|}{$\begin{array}{c}\text { Restricted Set } \\
\text { Unsupervised Configuration }\end{array}$} \\
\hline & Match & Mismatch & Match & Mismatch \\
\hline Diagonal-Left & 21 & 23 & 19 & 22 \\
\hline Diagonal-Right & 20 & 18 & 13 & 14 \\
\hline Vertical & 21 & 19 & 19 & 16 \\
\hline Horizontal & 21 & 20 & 20 & 18 \\
\hline
\end{tabular}

Thus, we conducted each analysis twice. First, we analyzed all subjects, regardless of performance. We then followed this up with a more conservative analysis which excluded subjects whose test data did not reflect differential responding to two categories. We computed the percentage of responses for each category at each location on the grad. We then excluded any subject who did not have at least 9 locations (of 52) for which category A was selected at least $50 \%$ of the time, and another 9 locations for which category B was selected at least $50 \%$ of the time. We didn't ask whether these locations were reasonable locations for each category - our goal was just to ensure that subjects responded reliably to two distinct categories. This resulted in the exclusion of 22 subjects yielding a sample of 141 (Table 1).

\section{Training}

Descriptives and Approach. Figure 3A shows accuracy over trials (binned in blocks of 40), and the category configuration in supervised training. There were clear effects of configuration, but not in a way that was predicted by COVIS: the two RB configurations showed similar performance, but performance was better in the diagonal-left condition (II), and worse in the diagonal-right. Figure 3B shows the same for the restricted set of subjects and shows a similar pattern, although the vertical group is now performing similarly to diagonal-left.

Figure 4A shows performance as a function of the match between unsupervised and 
supervised learning for each of the four configurations (which we term unsupervised-match). It suggests an effect of match, but only when for the diagonal-left configuration. Figure 4B shows the same for the restricted set of subjects. It shows continued evidence of a match benefit for the diagonal-left configuration, but now this is also seen for the other II configuration (diagonalright), and the other two configuration show no effect or a slight reversal.

Data were analyzed with a series of logistic mixed effects models implemented with the lme4 package (version 1.1-23), and the optimx package (version 2020-4.2)in R (version 4.02). The dependent variable was accuracy on each trial, and fixed effects include trial number, supervised boundary, and unsupervised-match. Supervised boundary was coded as three contrast codes that were motivated by the COVIS framing: the first contrasted the two RB configurations (+5., horizontal and vertical) and the two II configurations (-.5, diagonal-left and diagonal-right); the second contrasted the two RB categories (horizontal: +.5 ; vertical: -.5 ); the last contrasted the two II categories (diagonal-left: +.5 ; diagonal-right: -.5 ). Unsupervised-match was coded as match $=+.5$, mismatch $=-.5$. Because we had no a priori assumptions about the shape of the learning curve, prior to constructing the final model, we compared $1^{\text {st }}, 2^{\text {nd }}$ and $3^{\text {rd }}$ order polynomials of trial number, and found that adding both $2^{\text {nd }}\left(\chi^{2}(9)=75.8, p<.001\right)$ and $3^{\text {rd }}$ order terms $\left(\chi^{2}(9)=53.5, \mathrm{p}<.001\right)$ were significantly better than linear alone. Thus trial was coded as three orthogonal polynomials (centered and scaled to $+/-.5$ ).

The only possible random effects were the trial number terms. Following Seedorff, Oleson, and McMurray (submitted), we compared models with a random intercept of subject to one with a random slope of all three trial slopes and found that the fully sloped model offered a lower AIC (intercept only: AIC=75800, slopes: AIC=75452). Thus the final model was given in (1). Covariance terms were dropped to facilitate convergence. 


$$
\begin{aligned}
& \text { Correct } \sim\left(\text { trial }+\operatorname{trial}^{2}+\operatorname{trial}^{3}\right) * \text { UnsupMatch } *(\text { BoundaryRB }+ \text { BoundaryWinII }+ \\
&\text { BoundaryWinRB })+\left(\text { trial }+\operatorname{trial}^{2}+\operatorname{trial}^{3} \| \text { Subject }\right)
\end{aligned}
$$

With a model of this complexity it was not possible to report the complete results in the text. This is provided in Supplementary Note S1, and we focus on the most important results here.

Results: All Subjects. The intercept was highly significant (B=1.46, $\mathrm{SE}=0.06, \mathrm{Z}=25.07$, $\mathrm{p}<0.0001)$; as all fixed effects were centered this, confirms that mean performance was above chance (0.50). While the linear effect of trial was not significant $(B=-0.10, S E=0.10, Z=-1.01$, $\mathrm{p}=0.31)$, the quadratic $(\mathrm{B}=-0.39, \mathrm{SE}=0.17, \mathrm{Z}=-2.24, \mathrm{p}=0.02497)$ and cubic $(\mathrm{B}=3.10, \mathrm{SE}=0.53$, $\mathrm{Z}=5.83, \mathrm{p}<0.0001)$ were. This reflects the rapid rise in performance and suggests that any moderation of the rate of learning will occur on these terms (rather than the linear effect). Consistent with Figure $3 \mathrm{~A}$, the $\mathrm{RB} / \mathrm{II}$ contrast was not significant $(\mathrm{B}=0.13, \mathrm{SE}=0.12, \mathrm{Z}=1.16$, $\mathrm{p}=0.25)$, nor was the contrast within the $\mathrm{RB}$ configurations $(\mathrm{B}=-0.25, \mathrm{SE}=0.16, \mathrm{Z}=-1.53$, $\mathrm{p}=0.13)$. However, the contrast between diagonal-left and -right was highly significant $(\mathrm{B}=0.54$, $\mathrm{SE}=0.16, \mathrm{Z}=3.29, \mathrm{p}=0.00101$ ) with better performance in diagonal-left. There were no significant interactions between the three boundary factors and the trial factors (all $\mathrm{p}>.1$ ), though the within $\mathrm{RB}$ contrast had a moderate interaction with the quadratic effect of trial $(\mathrm{B}=0.83$, $\mathrm{SE}=0.49, \mathrm{Z}=1.68, \mathrm{p}=0.09376$ ). The lack of interactions again confirm no substantive difference between the RB and II categories; and suggest that the difference within the II boundaries was detected as an overall performance gain, not a gain in the rate of learning.

There was no overall effect of unsupervised-match $(\mathrm{B}=0.04, \mathrm{SE}=0.12, \mathrm{Z}=0.37, \mathrm{p}=0.71)$, nor did it interact with any of the trial factors (all p>.7). Unsupervised-match did not interact directly with any of the three boundary factors. However, we did find interactions of unsupervised-match, the supervised configuration and trial: We found significant three way interactions of unsupervised-match, the within II contrast, and the linear and cubic effects of trial 

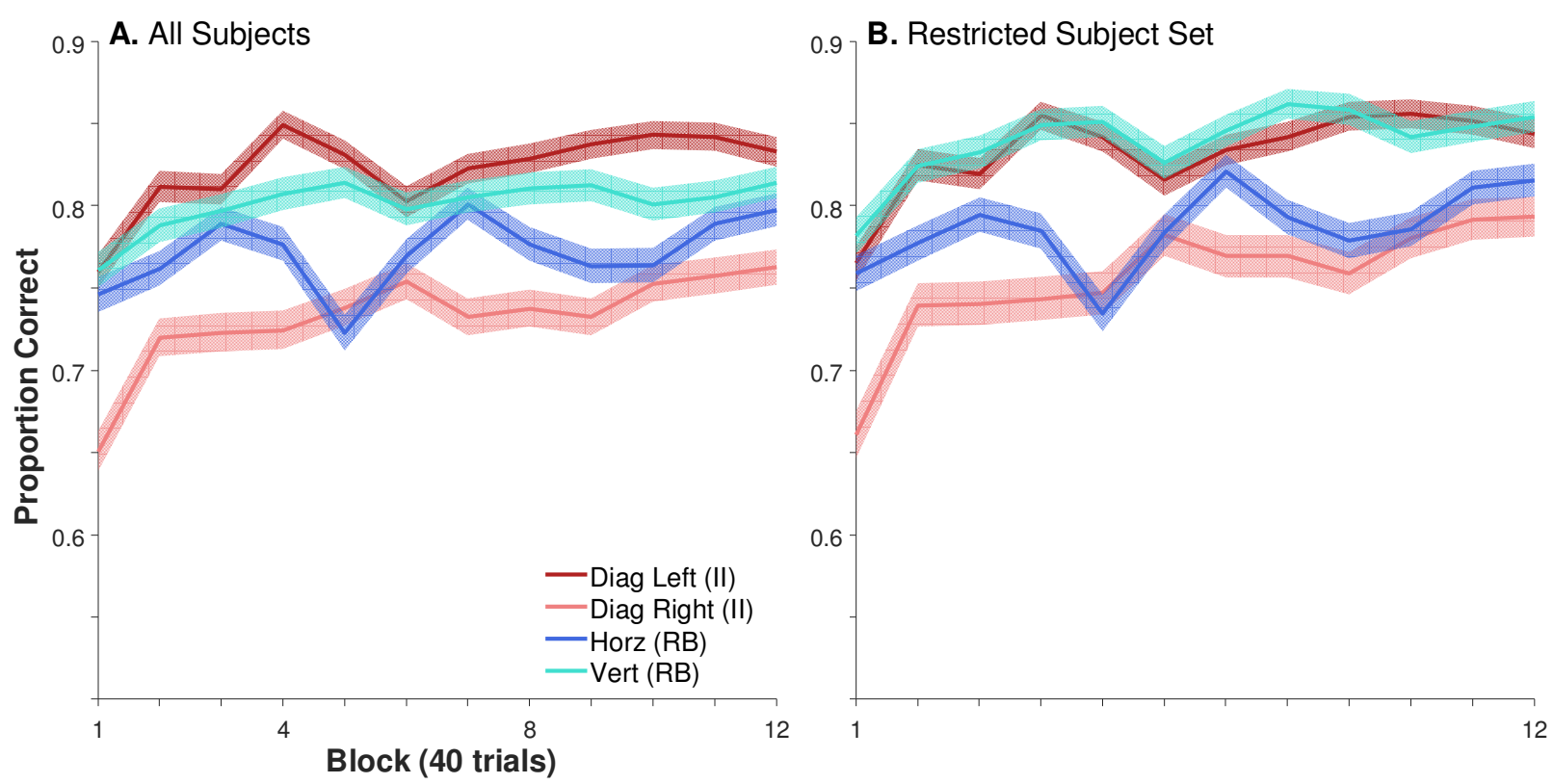

Figure 3: Accuracy as a function of training block and category configuration during the supervised learning phase. A) All subjects; B) restricted set. Shaded region is standard error of the mean.

(trial: $\mathrm{B}=-1.21, \mathrm{SE}=0.54, \mathrm{Z}=-2.25, \mathrm{p}=0.0248 ; \mathrm{B}=6.74, \mathrm{SE}=2.92, \mathrm{Z}=2.31, \mathrm{p}=0.0210$ ), and of unsupervised-match, the RB/II contrast, and the quadratic effect of time $(\mathrm{B}=1.43, \mathrm{SE}=0.69$, $\mathrm{Z}=2.08, \mathrm{p}=0.03728$ ). Working through the coefficients, this confirms the benefit of the unsupervised match for the diagonal-left categories, but largely at the middle blocks.

Results: Restricted Set. We next replicated this analysis with the more restricted set of subjects (Figure 3B, 4B) using an identical model to (1). Results were similar, though the overall pattern was somewhat simpler. Again there was no linear effect of trial, thought the quadratic $(\mathrm{B}=-0.57, \mathrm{SE}=0.20, \mathrm{Z}=-2.90, \mathrm{p}=0.00374)$ and cubic $(\mathrm{B}=2.97, \mathrm{SE}=0.59, \mathrm{Z}=4.99, \mathrm{p}<0.0001)$ were significant. This time there was a marignally significant $\mathrm{RB}$ vs. II contrast $(\mathrm{B}=0.20, \mathrm{SE}=0.11$, $\mathrm{Z}=1.77, \mathrm{p}=0.07599$ ), with $\mathrm{RB}$ categories performing a slightly better. However, the contrasts within each class were much larger: diagonal-left showed better performance than diagonal-right (as seen before; $\mathrm{B}=0.47, \mathrm{SE}=0.16, \mathrm{Z}=2.93, \mathrm{p}=0.00344$ ), and now the vertical configuration led to better performance than horizontal $(\mathrm{B}=-0.39, \mathrm{SE}=0.15, \mathrm{Z}=-2.53, \mathrm{p}=0.01143)$ (see Figure 3B). 

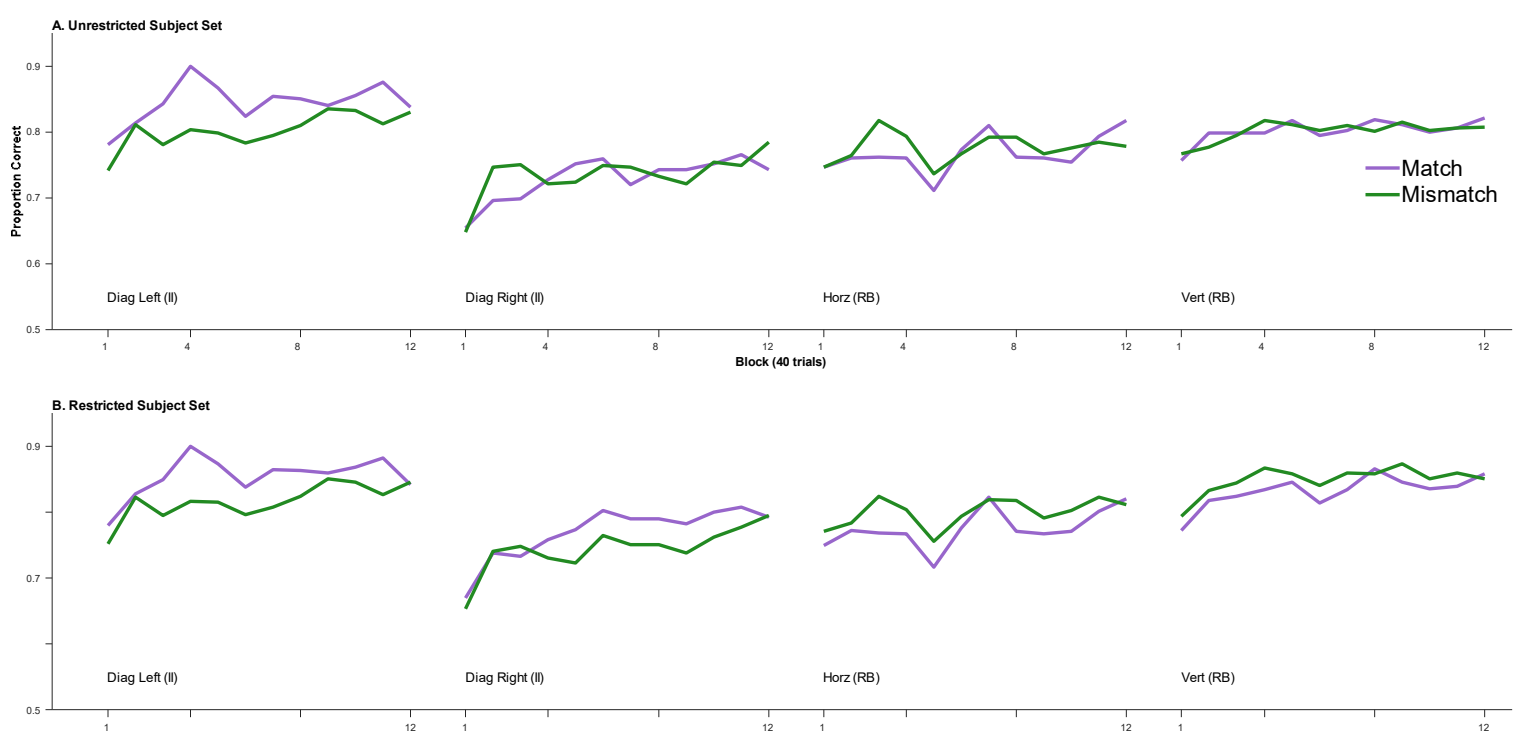

Figure 4: Accuracy as a function of training block (40 trials), supervised configuration, and supervised/unsupervised match. A) All subjects. B) Restricted set.

Thus, differences between configurations within a class again outweighed differences among the classes. As before, configuration did not interact with any of the trial number variables.

As in the prior analysis, there was no overall effect of unsupervised-match $(B=0.04$, $\mathrm{SE}=0.11, \mathrm{Z}=0.38, \mathrm{p}=0.70$ ), and it did not interact with trial. However, we found a three-way interaction of unsupervised-match, $\operatorname{trial}^{2}$ and the $\mathrm{RB}$ vs. II contrast $(\mathrm{B}=1.65, \mathrm{SE}=0.79, \mathrm{Z}=2.10$, $\mathrm{p}=0.03616$ ). This was the only interaction with unsupervised-match. In the prior analysis, this interaction was observed along with two three-way interactions among the within-RB and within-II contrasts, as only the diagonal-left boundary showed an effect of unsupervised-match. Here, the lack of such interactions supports the finding that the effect of unsupervised-match is broadly observed in both of the II boundaries (diagonal-left and diagonal-right, Figure 4B).

Dicussion. These analyses reveal several key findings. First, the pattern of performance was not broadly consistent with COVIS: there was little evidence for a systematic benefit for RB configurations. Instead, the difference in performance across the four configurations may derive 
from auditory factors. In particular, the pattern of results suggests listeners were not treating the two tones as independent cues, but rather that part of their performance was driven by a single cue that combines both (e.g., that the Tone 1 and Tone 2 frequencies are integral). In the diagonal-left categories (for example), the relevant dimension (the dimension orthogonal to the boundary) is one in which both cues are correlated: a given exemplar belongs to category A if both Tone 1 and Tone 2 are low frequency, and it is in category B if they are both high. In contrast, in the diagonal-right condition, the cues work in opposition: the exemplar is in category A if Tone 1 is low but Tone 2 is high. If listeners come to the task assuming only a single cue (e.g., mean frequency of both tones), this can explain why diagonal-left showed the best performance, and diagonal-right the worst. This combined cue would only be partially helpful for vertical and horizontal categories, predicting intermediate performance- exactly what was seen. The fact that the vertical categories are better than the horizontal suggests Tone 1 may have more weight in this combined value (perhaps due to upward masking).

Second, we did not find an overall effect of unsupervised-match. Rather, this effect was limited to particular configurations. In the analyses of all subjects, it was only seen in the diagonal-left configuration, while with the more restricted set it was seen in both the diagonal (II) configurations. We see two possible interpretations. First, if both configurations are amenable to unsupervised learning, this might be interpreted as evidence that only II categories show unsupervised learning (contradicting COVIS). Alternatively, it may be that unsupervised learning is most effective in situations where the cues are assumed to be correlated (or anticorrelated). That is, unsupervised learning may help sort out how cues are integrated. This can be addressed by the test trials. 

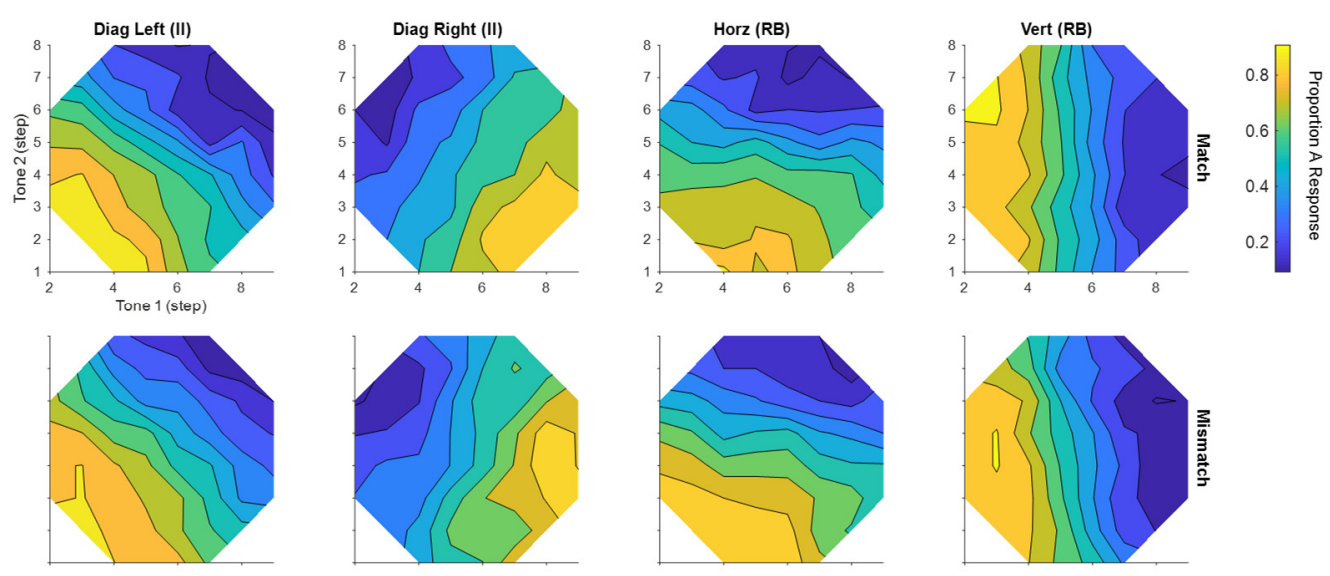

Figure 5: Results of test trials. $\mathrm{X}$ axis is tone 1 frequency; $\mathrm{Y}$ axis is Tone 2. Color represents likelihood of choosing category A (yellow) or B (blue). Column refers to the supervised configuration. Top row is unsupervised match; bottom row is mismatch. Data derived from all subjects.

\section{Test Trials}

Descriptives and Approach. On test trials, subject heard a range of stimuli uniformly distributed across the acoustic space (Figure 2F,G). This was done to determine if the structure of the category (in the full 2D space) differed as a function of configuration and unsupervised learning. Figure 5 displays these results as contour plots (for all subjects). The $\mathrm{X}$ and $\mathrm{Y}$ axes correspond to Tone 1 and Tone 2, and the darkness/blueness shows the degree to which Category A was selected. The top row (Figure 5A-D), shows the unsupervised-match groups, the bottom (5E-F) shows mismatched groups. In general participants, learned the appropriate boundary for their configuration, and showed relatively flat performance within categories. In contrast to these group-level results, Figure 6 shows plots for representative individuals. It shows marked variability, with some subjects acquiring the wrong boundary or a nonlinear boundary. Thus, many individual subjects may not have fully learned a stable category structure.

Our plan was to analyze these in a mixed model with each tone frequency as fixed factors along with category configuration and unsupervised-match. However, given the inherent differences in the orientation of the boundary between groups, it was clear that the configuration 

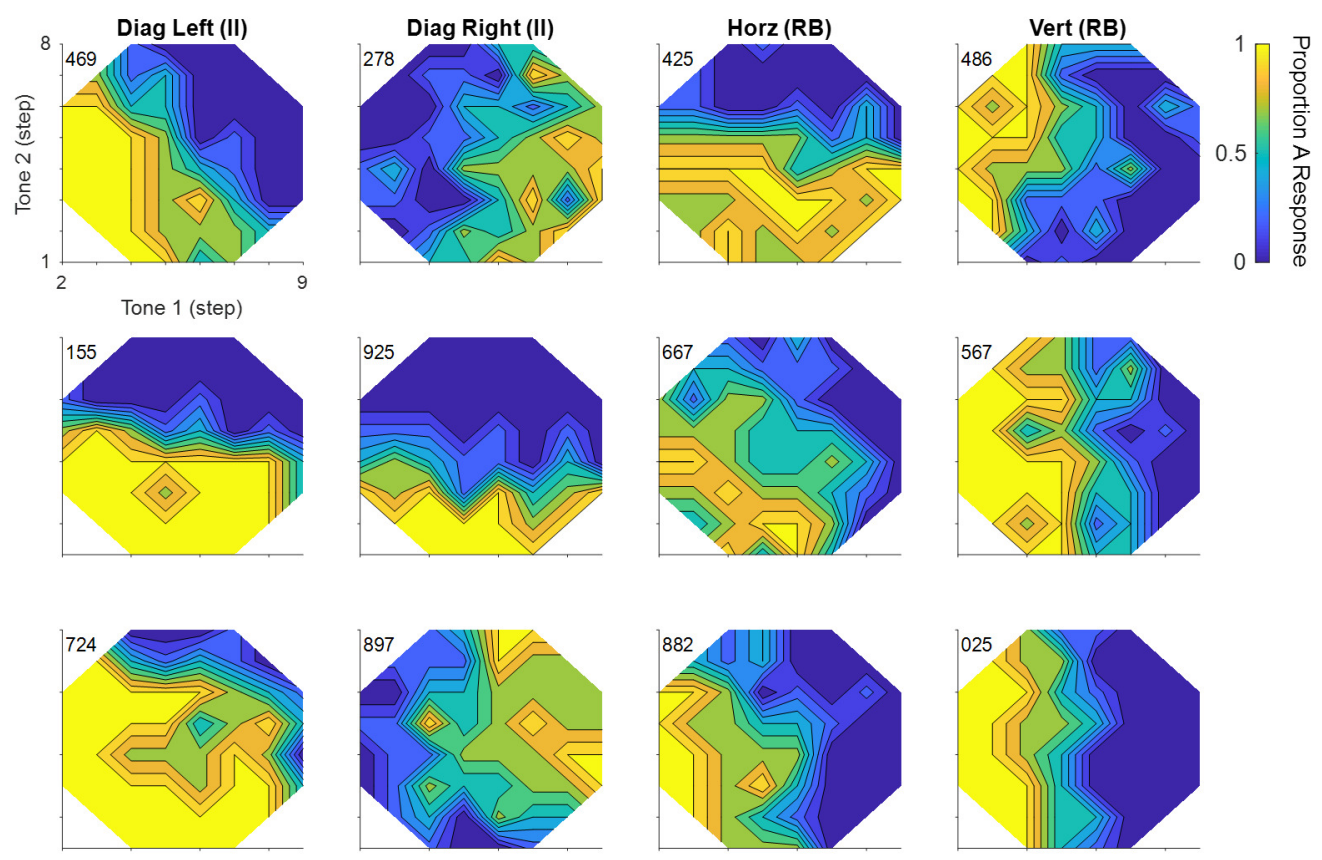

Figure 6: Performance on test trials for representative individual subjects.

$x$ tone interactions would carry most of the variance. This could make it hard to see systematic differences as a function of the particular boundary (e.g., if horizontal were steeper than vertical), and it may be difficult to see small differences (e.g., a moderation of that two-way interaction by unsupervised learning), particularly if they were specific to a configuration (e.g., if the slope of the categorization function was shallower for the diagonal-left than other configurations).

Thus, we started by rotating the auditory space to align it relative to each subjects' taught boundary. For each condition, we first computed the deviation $(\theta)$ between the angle if the boundary in that condition a 90 degree (vertical) boundary. We then used standard linear algebra formulas to rotate all of the Tone $1 \times$ Tone 2 coordinates of the grid by that amount (Equations 2,3). In this equation, Tone 1 and 2 are the step numbers along the test matrix. The result of this transformation was one dimension that was now perfectly orthogonal to the boundary-the "relevant" dimension—and a second dimension that ran parallel to the boundary- the "irrelevant" dimension. We then replaced the orignial Tone 1 and Tone 2 coordinates in the test 
data with the rotated coordinates for that condition.

$$
\begin{aligned}
& \text { Relevant Dimension } \\
& \text { Irrelevant Dimension }
\end{aligned}
$$

We examined the proportion of responses to Category A as a function of each dimension. We asked two questions. First, when plotted along the relevant dimension, this forms a sigmoidal response function (as in categorical perception paradigms). Here, if a particular configuration or unsupervised-match condition leads to sharper categories, we should see steeper slopes along the relevant dimension. Second, if subjects' boundaries were perfectly aligned to the relevant dimension, they should ignore the irrelevant dimension and performance should be flat at 0.5 . Thus, the extent that this function is not flat in one or more conditions suggests that these conditions make it challenging to properly weight and combine cues.

Figure 7 shows categorization along the relevant dimension. Figure 7A shows moderate differences among the configurations (ignoring unsupervised-match). Like in training, these differences do not pattern with the RB/II distinction: the diagonal-right configuration had the shallowest slope, and diagonal-left had the steepest, and vertical and horizontal were in between. Instead, this may better pattern as a function of the correlation between the two tone frequencies, as Tone 1 and Tone 2 were correlated for the best performing diagonal-left, but anti-correlated in worst performing diagonal-right (and uncorrelated for vertical and horizontal). Figure 7B and 7C show the overall effect of unsupervised-match averaged within the two II categories (Figure 7B) and RB (Figure 7C). It shows very little effect of the unsupervised distribution on the structure of the resulting categories for either class of boundaries.

Figure 8 shows the same results broken down by irrelevant dimension. Here we saw marked differences between configurations. The best-performing configuration, diagonal-left (Figure 8A), was mostly flat, implying that participants correctly ignored the irrelevant 


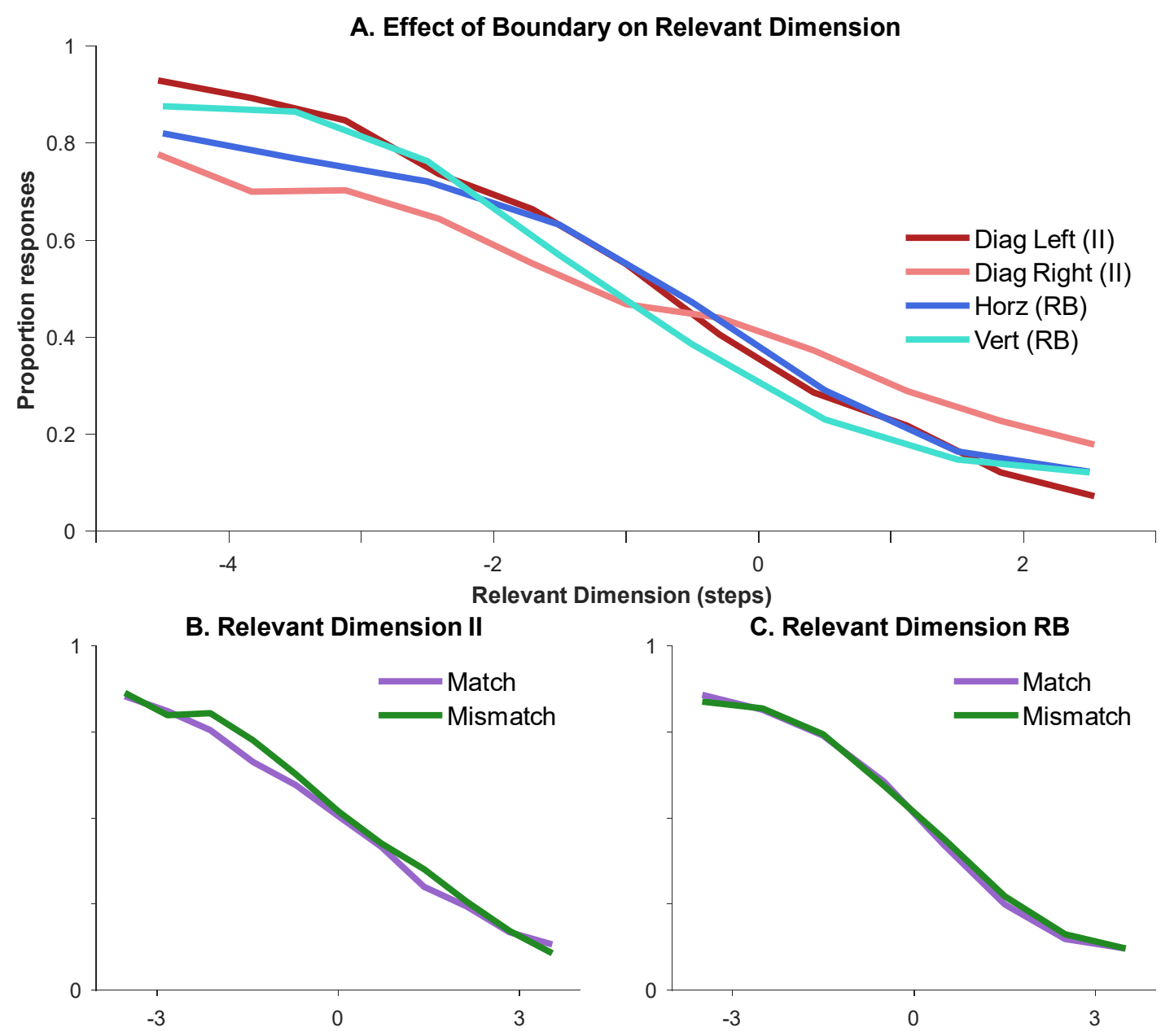

Figure 7: Testing results (after rotating the coordinate space) for all subjects. Each curve shows the proportion of Category A responses as a function of the relevant dimension. A) Effect of supervised configuration. B) Effect of unsupervised/supervised match for the two II supervised configurations (diagonal-left and diagonal-right). C) Effect of unsupervised/supervised match for the two RB configurations (vertical and horizontal)

dimension. In contrast, the other three category configurations (Figure 8B-D) showed sloping patterns, implying that subjects erroneously used the irrelevant dimension. In fact, working through the details of the rotation procedure (see Supplementary Note S2 for figures and the analysis) these patterns_-including the reverse effect in the horizontal condition-were derivable from the hypothesis that Tone 1 and Tone 2 were not separable. Thus, for analysis, we reversed the sign of the irrelevant dimension for the horizontal configuration, essentially equating the 

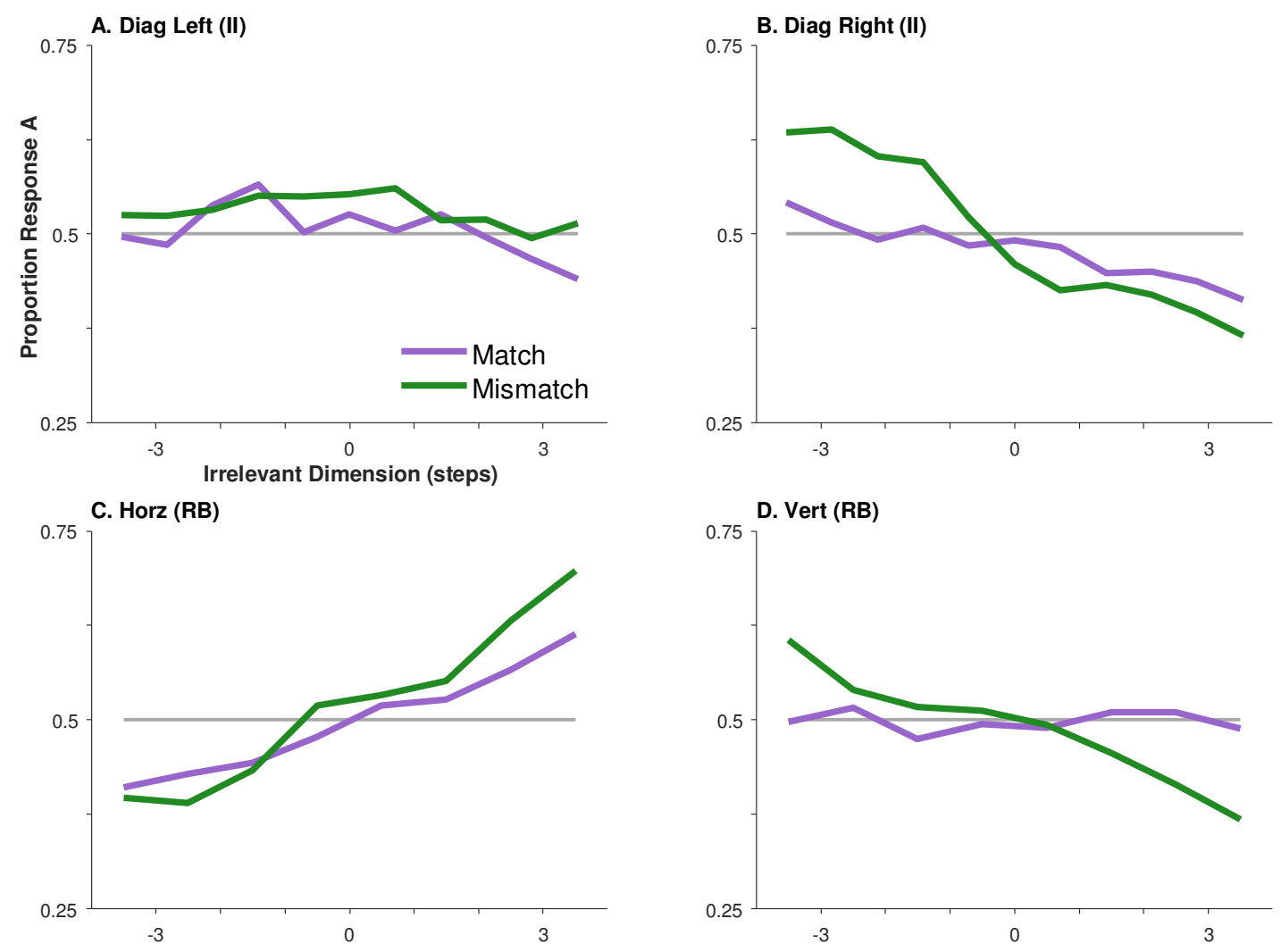

Figure 8: Proportion response A as a function of the irrelevant dimension (after rotation), and the supervised/unsupervised match. A) For the diagonal-left supervised boundary; B) for the diagonal-right boundary; C) for the horizontal boundary; D) for the vertical boundary.

predicted direction of the irrelevant cue effect across categories.

These rotated data were analyzed in a logistic mixed effects model with the following fixed effects: 1) configuration (the same three factors from the training analysis); 2)

unsupervised-match; 3) relevant dimension (centered and scaled to -1 to 1); 4) irrelevant dimension (centered and scaled); and 5) all of their interactions. In addition, exploratory analyses suggested that the irrelevant dimension may be non-linear, so we added an orthogonal quadratic term to capture this.

Finally, we were primarily interested in between-subjects factors (the effect of boundary and unsupervised-match). However, in a logistic mixed effects model between-subjects factors are evaluated against variance computed from trial-by-trial data. This can make it difficult to 
detect such effects if there is systematic trial-by-trial variance that is unaccounted for ${ }^{3}$. Thus to increase sensitivity we added two within-subjects factors to account for a recently discoveredand very strong-influence on performance in categoration tasks. In the presence of uncertainty (which is particularly acute here as many of the tokens are not near the boundary and there is no feedback), the stimulus and response on prior trials can shape performance on the current trial (Lieder et al., 2019). Specifically, if the present stimulus is acoustically close to that on the prior trial, subjects are likely to repeat the same response; however, if it is acoustically distant (even if in the same category), subjects are more likely to switch responses (see Supplementary Note S4.1 for an example of the strength of this pattern in the present data). Thus, we added the acoustic "distance" between the current stimulus and the last token (centered, scaled to -1/1); the subject's response on the last trial; and their interaction as covariates.

The relevant and irrelevant dimensions as well as these covariates were within-subject factors, and potential random effects. Again, we examined all possible random effects structure, and chose the one with the lowest AIC. This was the maximal model with slopes for everything. No covariance terms were included to facilitate convergence. The final model is given in (4).

$$
\begin{aligned}
\text { ResponseA } ~ & (\text { BoundaryRB + BoundaryWinII + BoundaryWinRB }) * \\
& \text { Relevant* }\left(\text { Irrelevant }+ \text { Irrelevant }{ }^{2}\right) * \text { UnsupMatch }+ \\
& \text { LastDistance * Last Response }+ \\
& \left(\text { Relevant } *\left(\text { Irrelevant }+ \text { Irrelevant }{ }^{2}\right)+\right. \\
& \text { LastDistance * Last Response II Subject })
\end{aligned}
$$

Results: All subjects. Complete statistical results of the model are provided in Supplementary Note S3 - we focus on the most important results here. Four crucial findings emerged from this model. First, the covariates showed the expected pattern: there was no main effect of either the last response $(\mathrm{p}=0.42)$ or the last stimulus distance $(\mathrm{p}=0.15)$, but the

\footnotetext{
${ }^{3}$ This variance would be averaged away in a standard GLM, but which cannot be in logistic regression which requires trial by trial data.
} 
interaction was highly signficant $(\mathrm{B}=-2.92, \mathrm{SE}=0.22, \mathrm{Z}=-13.23, \mathrm{p}=<0.0001)$. This is shown in Supplementary Note S4.1 where there is about a $20 \%$ difference in response rate. If the last stimulus was close to the present one, subjects were $20 \%$ more likely to chose the same category as they chose the last time; if it was far, they were $20 \%$ more likely to switch. This validates these covariates.

Second, we found a highly significant effect of the relevant dimension $(B=-6.43$, $\mathrm{SE}=0.31, \mathrm{Z}=-20.42, \mathrm{p}=<0.0001)$ with decreasing Category A responses as the relevant dimension increased. This did not interact with the RB / II contrast $(\mathrm{B}=-0.81, \mathrm{SE}=0.62, \mathrm{Z}=-1.30$, $\mathrm{p}=0.19)$, but it did interact with the within- $\mathrm{RB}(\mathrm{B}=1.90, \mathrm{SE}=0.89, \mathrm{Z}=2.14, \mathrm{p}=0.032)$, and within II $(B=-2.72, S E=0.88, Z=-3.10, p=0.002)$ contrasts. As in Figure 7, the vertical configurations (relying on Tone 1) showed steeper slopes than the horizontal (Tone 2); and the diagonal-left configurations (where Tone 1 and Tone 2 were correlated) showed steeper slopes than the diagonal-right (anti-correlated). As seen in Figure 7B,C, unsupervised-match did not interact with the relevant dimension $(\mathrm{B}=-0.07, \mathrm{SE}=0.62, \mathrm{Z}=-0.11, \mathrm{p}=0.91)$, nor were there any three way interactions of unsupervised-match, the relevant dimension, and configuration (all $\mathrm{p}>.25$ ). Thus, steepness of categorization along the relevant dimension was moderated by the configuration (though not in a way predicted by COVIS), but unaffected by unsupervised learning.

Third, there was an overall main effect of the irrelevant dimension on the linear scale $(\mathrm{B}=-1.01, \mathrm{SE}=0.30, \mathrm{Z}=-3.33, \mathrm{p}=0.0009)$ with a slight downward trend (Figure 8 ). This was not moderated by configuration (all $\mathrm{p}>0.3$ ). While the quadratic effect was not significant overall $(\mathrm{B}=-0.28, \mathrm{SE}=0.28, \mathrm{Z}=-1.00, \mathrm{p}=0.32)$, it was moderated by both the $\mathrm{RB}$ vs. II contrast $(\mathrm{B}=1.38$, $\mathrm{SE}=0.55, \mathrm{Z}=2.51, \mathrm{p}=0.012)$ and the within II contrast $(\mathrm{B}=-1.76, \mathrm{SE}=0.79, \mathrm{Z}=-2.24, \mathrm{p}=0.025)$, with higher order interactions of the relevant and irrelevant contrasts. Thus there may have been 
some nonlinearities in the use of the irrelevant dimension for some categories.

Finally, and most importantly, we examined the interactions of unsupervised-match and the irrelevant dimension. There was no overall interaction $(\mathrm{B}=0.89, \mathrm{SE}=0.60, \mathrm{Z}=1.48, \mathrm{p}=0.14)$. However we found strong four way interactions between the Irrelevant ${ }^{2}$ effect, and the unsupervised-match, relevant dimension, and both the RB/II contrast $(B=-7.90, S E=3.97, Z=-$ 1.99, $\mathrm{p}=0.047)$, and the within $\mathrm{RB}$ contrast $(\mathrm{B}=10.65, \mathrm{SE}=5.30, \mathrm{Z}=2.01, \mathrm{p}=0.044)$. As seen in

Figure 8, this reflects the fact that when unsupervised learning matched the supervised configuration, the irrelevant dimension played less of a role (the curve was flatter). The two interactions with boundary type reflects the fact that this effect was not present for the diagonalleft configuration—where the irrelevant dimension was not used regardless of the supervised learning. This further interacted with the relevant dimension (making it a four-way) because the steepening of the irrelevant effect was heightened at high levels of the relevant dimension (see Supplementary Note S4.2 for a visualization of this effect).

Results: Restricted Set. We next ran the identical model on the restricted set of subjects. Complete results are given in Supplementary Note S3.2, and corresponding figures are in S4.3 and S4.4. In general, results were similar. As before, we found a strong interaction of the prior response and the acoustic distance between the current and prior stimulus $(B=-3.00, S E=0.25$, $\mathrm{Z}=-12.17, \mathrm{p}=<0.0001)$. We also replicate the interactions between the relevant dimension and the boundary type (Relevant $\times$ w/in II: $B=-1.81, \mathrm{SE}=0.85, \mathrm{Z}=-2.13, \mathrm{p}=0.033$; Relevant $\times$ w/in $\mathrm{RB}: \mathrm{B}=2.70, \mathrm{SE}=0.81, \mathrm{Z}=3.34, \mathrm{p}=0.0009)$. As before the irrelevant dimension had an overall main effect $(\mathrm{B}=-1.23, \mathrm{SE}=0.35, \mathrm{Z}=-3.48, \mathrm{p}=0.0005)$, and the quadratic effect was moderated by the $\mathrm{RB}$ vs. II contrast $(\mathrm{B}=1.70, \mathrm{SE}=0.62, \mathrm{Z}=2.76, \mathrm{p}=0.006)$, and by the within $\mathrm{II}$ contrast $(\mathrm{B}=-$ 1.94, $\mathrm{SE}=0.92, \mathrm{Z}=-2.12, \mathrm{p}=0.034)$. Thus, the general pattern with respect to the effect of 
boundary type is confirmed.

With respect to unsupervised-match, effects were weaker and patterned slightly differently. As before, there was no interaction of unsupervised-match and relevant dimension $(\mathrm{B}=0.08, \mathrm{SE}=0.59, \mathrm{Z}=0.14, \mathrm{p}=0.89)$, nor did this interact with configuration (all $\mathrm{p}>0.2)$. This time there was a marginal interaction of unsupervised-match and the irrelevant ${ }^{2}$ effect $(B=-1.08$, $\mathrm{SE}=0.62, \mathrm{Z}=-1.75, \mathrm{p}=0.080$ ), and there was a marginal interaction of unsupervised-match, relevant and irrelevant $(B=-1.56, \mathrm{SE}=0.92, \mathrm{Z}=-1.70, \mathrm{p}=0.090)$. The unsupervised-match $\times$ relevant $\times$ irrelevant $^{2} \times \mathrm{RB} / \mathrm{II}$ contrast was also marginal $(\mathrm{B}=-8.74, \mathrm{SE}=4.93, \mathrm{Z}=-1.77, \mathrm{p}=0.077)$ (it was significant with all subjects), but the same interaction with the within/RB contrast was not $(\mathrm{B}=12.09, \mathrm{SE}=7.97, \mathrm{Z}=1.52, \mathrm{p}=0.13)$. These marginal effects must be interpreted with caution (if at all), but they suggest that effects were weaker in the restricted set, and the effect of unsupervised-match may have been limited to the II configurations.

Discussion. The results of the testing trials suggested several key findings. First, like the training trials, we see clear differences among the different configurations (Figure 7). These differences do not pattern strongly with RB / II distinction, but fit with the idea of partially integral dimensions. The diagonal-left categories relied on correlated cues. It showed the most robust categorization (a steeper slope on the relevant dimension), and the most precise use of cues (the lack of effects on the irrelevant dimension). In contrast, the diagonal-right configuration relied on anti-correlated cues and had the least robust, and the least precise, categorization. The vertical and horizontal configuration were somewhat in the middle (since the two cues were uncorrelated), with slightly better performance for vertical which suggests people may be biased be the lower frequency Tone 1 more than Tone 2 .

Second, we found no evidence that unsupervised learning affects steepness along the 
relevant dimension (Figure 7). Rather, the unsupervised learning appears to affect the way that listeners learn to ignore (or use) the irrelevant dimension. When the unsupervised distribution matched the supervised, unsupervised learning appeared to reduce the reliance on the irrelevant dimension. This effect was small, and only marginally signficant in the restricted analysis so it should be intepreted with caution. However, it is notable that it was limited to the three configurations in which identifying which cues are relevant and irrelevant is the most challenging. In the diagonal-left configuration, Tone 1 and 2 are correlated and work together. This appears to be the expectation that listeners brought to the experiment, and therefore unsupervised learning played a minimal role. The other three configurations violated these expectations and here we saw the strongest effects of unsupervised learning with flatter functions when it matched the configuration than when it mismatched.

\section{General Discussion}

Our discussion is framed in two parts. First, we'll discuss the immediate implications of the empirical work to better situate our findings in the literature on category learning. Next we return to the broader developmental issues that motivated our hybrid learning paradigm.

\section{Implications for Category Learning}

Our empirical study asked how prior unsupervised learning impacts later supervised learning. This was designed to mimic the ecology of category acquistion during L1 acquisition: children undergo a period of primarily unsupervised learning in infancy followed by later supervised learning as they gain access to a larger vocabulary, the ability to produce speech, and may undergo spelling instruction (which serve as implicit supervisory signals). 
In addition, there strong claims made by COVIS that different category configurations may be more easily learned (Ashby \& Maddox, 2011), and crucially, that unsupervised learning may only be possible for categories organized along a single dimension (RB) (Ashby et al., 2002b; Maddox et al., 2003). While the former claim has undergone extensive testing in auditory categorization (Chandrasekaran, Koslov, et al., 2014; Chandrasekaran, Yi, et al., 2014; Yi et al., 2016), the latter has not yet been evaluated. Given this, we crossed the unsuperivsedmatch with four category configurations (two II and two RB).

Category Configurations. We found that the RB or II configuration of the categories had almost no impact on performance. Rather, the diagonal-left configuration (II) was learned the best, but diagonal-right (also II) was learned the worst. Vertical and horizontal configurations were in the middle. Similar results were also seen in the steepness of the categories along the relevant dimension at test. This is not consistent with the basic predictions COVIS.

Instead, these results are consistent with the idea that the Tone 1 and 2 frequencies were not treated as fully independent, but rather at some level they were combined into a single dimension (something akin to pitch or spectral mean). This can explain why diagonal-left was learned the best (the tone frequencies were correlated), and diagonal-right was the worse (the listener had learn the opposite relationship). As we describe in Supplementary Note S2, this can also explain the specific pattern of utilization of the irrelevant dimension at test.

The fact that vertical configurations were generally learned better than horizontal enhance this explanation. In the vertical categories, the relevant dimension was Tone 1, while in Horizontal the relevant dimension was Tone 2. If the combined percept was weighted toward Tone 1, or if Tone 1 exerted some upward masking on Tone 2, this could give rise to this effect. At this stage it is not clear if the combined (and weighted) Tone 1/2 dimension is the only 
dimension that listeners considered, or if they also used each tone individually to some extent. However, either way it is clear that listeners came to the categorization problem with an assumption that these cues are not independent.

In a normal category learning experiment, integral cues are problematic - the researcher is supposed to ensure that the cues are separable before starting. However, our stimuli were motivated not as some idealized set of stimuli for understanding categorization, but from more ecological considerations. We sought to create non-linguistic stimuli (to avoid interference from the native language) that captured the kind of auditory dimensions that listeners face in the real world. In our case, these were modeled after vowels.

In fact, these kinds of correlations among cues are the norm in speech. As any introductory phonetics student can tell you, a quick glance at the vowel space of English (Hillenbrand et al., 1995; Peterson \& Barney, 1952) shows strong correlations between F1 and F2. Or consider consonant voicing in English. Voiceless sounds are associated with aspiration (instead of laryngeal voicing), as well as higher pitch and higher F1 frequencies (Ohde, 1984; Summerfield \& Haggard, 1977). However, aspirated segments have a higher spectral mean than than voiced, and thus, all of these can be partially described as higher frequency cues voiceless sounds. In fact, perceptual training studies suggest listeners cannot fully reverse or reweight these natural correlations between voicing cues (Francis, Kaganovich, \& Driscoll-Huber, 2008; Idemaru \& Holt, 2011). Moreover, integrality may be the norm in auditory perception: even arbitrarily related cues like center frequency, and amplitude modulation frequency often appear integral in category learning experiments (Holt \& Lotto, 2006).

Looking broadly at the phonetics literature, there are almost no distinctions characterized by isolated cues (Espy-Wilson, 1992; Hillenbrand et al., 1995; Lisker, 1986; McMurray \& 
Jongman, 2011), and it is not clear that phonetically cues are truly independent or separable. We may never be able truly identify a small number of separable cues for speech. If this is the case strong distinctions like the RB/II distinction posited by COVIS may be irrelevant for speech. Instead, all speech categories may require information integration, though some dimensions may be integral. As Francis, Kaganovich, et al. (2008) point out, a more relevant framing may be in terms of dimensional attention to sets of partially integral cues. However, it is important to point out that even in this framing, the present study suggests that integral dimensions in auditory categorization are not immutable - even in the diagonal-right configuration (which required listeners to reverse the relationship between Tone 1 and 2) listeners were still able to learn the categories (see also, Francis, Kaganovich, et al., 2008; Idemaru \& Holt, 2011). Rather integral cues may function more like strong priors on how the auditory space should be organized. These priors are present at the beginning of training, but can be overridden.

Unsupervised learning for all sorts of categories. We operationalized the presence of unsupervised learning as a gain in supervised learning when the unsupervised configuration matched the supervised (e.g., Mirman et al., 2008). While the effects of unsupervised learning were small (a point we come back to) we did not find evidence that unsupervised learning was not operational any specific configurations. On training trials, we found accuracy benefits primarily in the II configurations (Figure 4): in the full dataset this was for the diagonal-left category, and in the reduced dataset for both. However, at test we saw benefits in the RB configurations too: three of the four categories (all except diagonal-left) showed reduced utilization of the irrelevant dimension when unsupervised learning matched the supervised.

COVIS makes the strong claim that unsupervised learning should only be available to RB categories (Ashby et al., 2002a; Ashby et al., 1999). It is unclear how to evaluate this claim in 
light of the likely integrality of the cues: it's unclear at this point if any of these configurations can be truly classified as II or RB. If we take the dimensions at face value, it is clear that particularly with respect to training, the II configurations undergo unsupervised learning. Alternatively, if we treat the integrality of Tone 1 and Tone 2 as fundamental, then the diagonalleft is likely the best candidate for an RB configuration (since it uses this single integral dimension). But here we found strong evidence for unsupervised learning during training, but on the other hand it was the only category that showed no evidence of it at test (though as we argue later this may be the exception that proves the rule).

One possibility is simply that we lacked the power to truly test this claim - while there were 163 subjects overall, when we break this down into a 4 (configuration) $\times 2$ (unsupervised match) design, there are only roughly 20 subjects in each group (Table 1). It may be that there is a broader effect of unsupervised learning that affects all categories, but that we were unable to observe it systematically in this smallish sample. So we are hesitant to make strong claims about whether unsupervised learning is restricted to one type of category. But this argues more broadly that with respect to the applicability of unsupervised learning to particular category configuration, we shouldn't be worrying about the informational structure of the categories, but rather about the goals that unsupervised learning must achieve.

On the effectiveness of unsupervised learning. Despite these effects, it is also clear that unsupervised learning plays a small role relative to supervised learning. In the training data (Figure 4), the unsupervised-match benefit rarely exceeded 10\%, and it was limited to the II configuration. If listeners were really learning categories during the unsupervised phase, one would expect that much larger effects, since after unsupervised learning, the listener would only need to remap the implicit category labels from the unsupervised learning to the ones provided 
by supervised learning. This should have led to large gains, particularly at the beginning of training. In contrast when we found gains they were small and distributed throughout training.

Similarly, on the test trials, the unsupervised learning had no effect on the most important aspect of learning: steepness of the category along the relevant dimension (Figure 7B,C). The slope of the category structure (corresponding to the precision of the boundary) was not affected at all by the unsupervised learning. Instead small effects were observed on the utilization of the irrelevant dimension (Figure 8), though these were only observed for three of the four category structures (all but diagonal-left), and often were restricted to only part of the category space (only at higher values of the relevant dimension).

Of course, it was possible that our unsupervised exposure was simply insufficient to yield enough learning to drive such effects. However, we note that the number of unsupervised trials (300) was quite close to the number of supervised trials (384). And our best estimate of the course of learning (from the supervised trials) suggests very rapid learning within the first hundred or so trials (with diminishing returns later). Thus, even if additional unsupervised trials would have helped, minimally we can conclude that supervised learning clearly had a more powerful effect than unsupervised leearning. However, an alternative is that perhaps unsupervised learning is not about acquiring categories, but rather is doing something else that later contributes to supervised learning.

What is unsupervised learning accomplishing? The standard view of unsupervised learning is that learners are acquiring the categories (though they may lack explicit labels) (McMurray et al., 2009; Vallabha et al., 2007) - indeed this is somewhat necessary to account for the intuition that infants "have" the categories of their language by around 18 months (Werker \& Curtin, 2005). While the present data do not speak to infants, we don't see much 
evidence that learners have acquired anything like categories by the end of the unsupervised phase. So what else could unsupervised learning be accomplishing?

A critical point is that at test, unsupervised learning had little effect on the structure of the categories along the relevant dimension, but did help learners rule out the irrelevant. This suggests people may use unsupervised exposure to reshape the auditory space (precategorically). In the diagonal-right case, for example, they may be learning to reverse the relationship between cues; while in the vertical case, they may be learning to treat the cues independently (using Tone 1, but ignoring Tone 2). This then can explain why we see no benefit for unsupervised-match in the diagonal-left configuration: this configuration respects the integrality of the cues, so learners were making the right assumptions about the auditory space all along.

This restructuring is distinct from the warping posited by the perceptual magnet effect or categorical perception. In those views, the auditory space may be warped such that regions that correspond to categories are shrunk, and distances across the category boundary are enhanced. This has now been conclusively ruled out as a model of adult perception. The earlier evidence for such warping turnedout to be largely an artifact of task (Gerrits \& Schouten, 2004; Massaro \& Cohen, 1983; Schouten, Gerrits, \& Van Hessen, 2003), and listeners appear to track continuous cues gradiently (Toscano, McMurray, Dennhardt, \& Luck, 2010). In contrast, we propose something more like principle component analysis which collapses a high dimensional space down to a smaller number of dimensions while preserving the overall gradiency in the system. It is this transformed space that supervised learning then carves up into categories. If this is what is going on during unsupervised learning, why was there no effect of unsupervised- match on the relevant cue (Figure 7B,C)? We suspect that this is because learners are treating this as a multi-dimensional space, simultaneously considering each tone individually, 
the integral combination, the reverse combination and so forth. The supervised learning is uniquely suited to rapidly extracting the relevant cue (making it somewhat susceptible to ceiling effects); the unsupervised learning may play a role in helping to suppress secondary dimensions.

This kind of learning is not captured in any theoretical models of categorization. But, a few come close. SUSTAIN (Love et al., 2004), for example, has a pre-categorical representation that can be tuned by unsupervised learning. These take the form of clusters of features and are highly sensitive to the naturally occurring distributions in the environment. They are later mapped to category representations via supervised learning. Conceptually this captures much of what may be going on here with one exceptions: our work suggests that the pre-categorical presentations may not represent clusters but rather dimensions that cut across the input space.

Early connectionist models of unsupervised speech category learning offer some ideas as well (Gauthier et al., 2007; Guenther \& Gjaja, 1996). These models use self-organizing feature maps (Kohonen networks) to reorganize the input into a new map based on the statistics of the input. This map represents the dimensionality of the input in a more or less continuous space. This concept seems to fit with what we observe, with two key exceptions. First, in these models the clustering of regions in the map is thought to encapsulate categorization itself. That seems unlikely here - more likely is that this serves as the input space to a supervised learning system that actually learns the categories (though see Westermann \& Miranda, 2004). Second, because of this clustering, these models show evidence of phonemona like the perceptual magnet effect; however, this is inconsistent with the evidence that people's pre-categorical representation of speech cues is continuous, not warped (Toscano et al., 2010). It is also not consistent with the results here showing no effect of unsupervised learning on the steepness along the relevant dimension. Nonetheless, these model offer conceptual fit to the spirit of our proposal. 
Finally, Toscano and McMurray (2010) present a model in which cue values are weighted and linearly combined prior to categorization. In this model, weights are assigned during unsupervised learning, much like we propose here. In this case, cues that are more reliable (that have greater clustering) are given greater weight. This kind of architecture may be able to account for these effects. However, their (our) implementation may be too limited in that weight is only assigned based on within-cue statistical properties (the model largely ignores correlations between the cues) and does not (yet) include supervised learning. Nonetheless, the overall architecture is promising.

While there are a candidates for models to explain these effects, none fully capture the mechanisms that appear to be operative here: that listeners reorient the acoustic space during unsupervised learning so that it can be later parcelled up for supervised learning. Nonetheless, all of these models offer promising starts by arguing for a layer of auditory representation before categories are imposed, which can be remapped by unsupervised learning.

Methdological implications. Finally, as a new paradigm in auditory category learning, it is worth briefly mentioning a number of methodological issues. First, it is clear that unsupervised learning can be studied in this way. Since learners are never taught any explicit labels for the categories, prior unsupervised learning studies have been only to infer the results of learning indirectly via measures like discrimination (Escudero et al., 2011; Escudero \& Williams, 2014; Maye \& Gerken, 2000) or with meta-categorical sorting tasks (Goudbeek et al., 2008; Goudbeek et al., 2009). The reinforced supervised learning paradigm can overcome this by using a brief supervised phase to teach learners the labels. While this has the potential to mask any unsupervised learning, it also opens up new ways to assess it. In this way, it may be a useful complement to other paradigms. 
Second, the paradigm "works" in that it could clearly reveal the contributions of each phase of learning, as well as differences among different types of categories. However, effects of unsupervised learning were not strong. Clearly the nature of the cues (e.g., integral or separable) may play an important role in this that needs to be carefully considered in future work. There were also clear individual differences revealed at test (Figure 6). It may help to over-powering the test trials (more repetitions) if the goal is better characterize category leaerning at an individual level. Moreover, large numbers of subjects may be needed to detect systematic effects - here, online testing may be a boon.

Third, there are useful techniques here that other projects may adapt. Most importantly, rotation of the stimulus space before analysis is simple and can allow people to cleanly compare categories of different configurations. Our analysis of the test trials points to the need to capture systematic trial-by-trial variance (e.g., the last trial distance effect) for more sensitive measures.

Perhaps most importantly, this paradigm offers a number of important future directions to model different types of learning that may occur in the real world. For example, one can model aspects of L2 learning by introducing the categories initially via supervised training (e.g., the classroom), followed by unsupervised (immersion). Or one could model critical period effects as a form of pro-active interference by training the subjects on one structure followed by another.

\section{Implications for Language Development}

The initial motivation for unsupervised learning in the acquisition of speech categories was motivated by the ecology of learning (Maye, Weiss, et al., 2008; Maye et al., 2003; McMurray et al., 2009; Vallabha et al., 2007). It appeared that infants were acquiring the categories of their langauge well before they had access to any implicit teaching signals like a 
large lexicon, speech production, or spelling. This has been productive as it led to great insights about the variety of learning mechanisms that people can and do employ for language.

However, as our literature review reveals, our understanding of the ecology of speech category learning may be incomplete. It is now clear that speech categories continue to develop far late into life (Hazan \& Barrett, 2000; McMurray et al., 2018), and that unsupervised mechanisms are not limited to infancy or to the L1 (Escudero et al., 2011; Escudero \& Williams, 2014; Goudbeek et al., 2008; Goudbeek et al., 2009). This changes our picture of the ecology of learning — and this is what motivated the reinforced statistical learning paradigm proposed here.

At the broadest question, this line of thinking (and our empirical work) raises the question of whether infants are even learning speech categories? We cannot rule out that they they are, but we note that this rests of somewhat less solid evidence than we might think.

First, babies have never been tested in a pure identifcation task. Often times, we test discrimination, and infer that categorization must be present when within-category discrimination is poor, and between-category discrimination is high (e.g., Eimas et al., 1971; Werker \& Tees, 1984). However, this rests on an assumption that speech is percieved categorically (Liberman, Harris, Hoffman, \& Griffith, 1957), an assumption that has not withstood scrutiny as a model for adult perception (Gerrits \& Schouten, 2004; Massaro \& Cohen, 1983; Schouten et al., 2003; Toscano et al., 2010). If adults don’t exhibit categorical perception in well understood tasks, why should this assumption be justifed in for interpreting infant performance, particularly when this assumption relies on infant discrimination tasks that are even less well understood (Galle \& McMurray, 2014)! While some studies have adapted identification measures like conditioned head turn (Kuhl, 1979), or anticipatory eye-movements (AlbaredaCastellot, Pons, \& Sebastián-Gallés, 2011), these require infants to be trained on the 
categorization. And even if we take both the categorization and discrimination data at face value, often times infants are responding to a sequence of speech sounds, not a single one (as in a typical adult paradigm). So what it means for infants show above chance performance may be very different than the standards we hold adults to. Thus, while it is safe to assume that infants are adapting their auditory system to the language, there is little data that unamibiguously argues that that they actually have categories.

The present study offers hints of an alternative. It suggests that unsupervised learning is actually fairly weak and may simply serve to reorganize the perceptual space, the perceptual space that is later carved into categories by perhaps more implicitly supervised experiences. This is consistent with theoretical models like WRAPSA (Jusczyk, 1993), and with computational models like SUSTAIN (Love et al., 2004) (and see Gureckis \& Love, 2004, for a discussion of the relationship between infant and adult categorization tasks), Westerman's version of a self-organizing map (Westermann \& Miranda, 2004), and Toscano's weighting by reliability scheme (Toscano \& McMurray, 2010). It also helps rectify an important challenge raised by work in infant directed speech: some important speech categories simply don't separate into clear clusters (Bion et al., 2013), and in many ways, infant directed speech makes speech categories more confusable (Cristia \& Seidl, 2013; Martin et al., 2015; McMurray et al., 2013; Miyazawa, Shinya, Martin, Kikuchi, \& Mazuka, 2017). A hybrid model of this sort may help make sense of this - unsupervised learning alone is not responsible for speech category acquisition. Moreover, if the "goal" during infancy is simply to restructure the space, the enhanced variation often seen in infant directed speech could help highlight the covariation between potential cues. This argues—-much like we've simulated here—-that infant unsupervised learning may set a rough stage for later learning by helping to organize the auditory space; and 
later supervised learning may leverage sources of implicit feedback like lexical development, production, or spelling to actually acquire the categories.

We also need to acknowldge the limits of our account with respect to real-world acquisition. In the relatively simple sequence we've proposed here, unsupervised learning may only play one role - auditory reorganization. However, after categories have begun to be established by supervised learning, it may play further roles - it could help consolidate or refine these categories (and this of course, may of course be further altered by sleep). Similarly, supervised learning could play a powerful role in not just aligning to the auditory space to the statistics of the input, but also to the functional goals of the listener (e.g., the structure of the langauge) (Love \& Gureckis, 2005). Indeed McMurray et al. (2018) demonstrate that children and even adolescents are still developing their ability to retain and use-fine-grained continuous detail. Given that this is occurring during a time that implicit supervised learning is thought to be occurring, this suggests that it may also help organize the auditory space. These roles could potentially be isolated with further permutation of the sequence of events in the reinforced statistical learning paradigm.

One final question that this raises however, concerns L2 acquisition. If supervised learning is so much more powerful than unsupervised, and if L2 acquistion is properly thought of as primarily supervised, then why do adults struggle to learn new speech categories? We argue that this is not for lack of plasticity. The last two decades have shown an explosion of work on auditory learning and plasticity in adult listeners (e.g., Davis et al., 2005; Francis, Kaganovich, et al., 2008; Guediche, Fiez, \& Holt, 2016; Holt \& Lotto, 2006; Idemaru \& Holt, 2011; Norris et al., 2003) (see Samuel \& Kraljic, 2009, for an older review). While adults do not learn everything equally well, they show incredible plasticty and learn far more than we ever thought 
possible. Moreover, recent work by Fuhrmeister, Schlemmer, and Myers (2020) suggests that adults may actually be superior to children at learning non-native speech sounds.

Instead, we suspect this seemingly "sensitive period" is really a form of proactive interference. Adults learning an L2 already have a set of categories that span much of the acoustic space they may need to reconfigure for their L2 (and when these categories do not—as is the case with Zulu click sounds for English listeners- they find this much easier: Best, McRoberts, \& Sithole, 1988; Pisoni et al., 1982). This could make it harder to learn new categories. Much of the work on adult plasticity (not L2) has involved shifting existing speech categories (e.g., to account for a new talker, new pronunciations, etc)—here, existing categories are not being over-written, but merely adapted. This may create less interference than attempting to learn new categories that sub-divide or collapse existing ones. Thus, while it may be functionally true that adults have a more difficut time learning new categories, this may not derive from any biological changes (e.g., closing a window of plasticity), but rather from more basic properties of learning and memory. We suggest that paradigms like the reinforced statistical learning pardigm- in which learners are essentially exposed to two sets of mappings in a sequence - may be helpful in understanding this sort of interference that adult learners may confront when learning the categories of an L2.

\section{Conclusions}

At the broadest level, speech category acquisition requires multiple learning mechanisms, both supervised and unsupervised. This is supported by our emerging understanding of the development of speech categorization - the ecology of auditory learning - and by the empirical work we've shown here. These mechanisms may play different roles. We've proposed that 
unsupervised learning [at least initially] may primarily serve to reorganize the auditory space,

while supervised learning later carves it into categories. This is supported by the empirical

results from the reinforced statistical learning paradigm, which was configured to isolate this

particular sequence of events (as a simplification of L1 acquisition). However, the true

development of speech perception is likely to be more complex than this, as the functions of

these modes of learning may differ as a function of the developmental history of the learner.

\section{Acknowledgements}

The authors would like to thank John Freeman, Rodica Curtu, Ted Abel and McCall Sarrett for helpful comments during the development of this project; and Jason Gellar for assistance developing the online testing procedures. This project was supported by DC 0008089 awarded to BM; an Iowa Neuroscience Institute pilot grant awarded to BM; and an NSF Graduate Research Fellowship awarded to SLC.

\section{References}

Albareda-Castellot, B., Pons, F., \& Sebastián-Gallés, N. (2011). The acquisition of phonetic categories in bilingual infants: New data from an anticipatory eye movement paradigm. Developmental Science, 14(2), 395-401.

Ashby, F. G., \& Alfonso-Reese, L. A. (1998). A neuropsychological theory of multiple systems in category learning. Psychological Review, 105(3), 442.

Ashby, F. G., \& Maddox, W. T. (2011). Human category learning 2.0. Annals of the New York Academy of Sciences, 1224(1), 147-161.

Ashby, F. G., Maddox, W. T., \& Bohil, C. J. (2002a). Observational versus feedback training in rulebased and information-integration category learning. Memory \& Cognition, 30(5), 666-677. doi:10.3758/bf03196423

Ashby, F. G., Maddox, W. T., \& Bohil, C. J. (2002b). Observational vs. feedback training in rule-based and information-integration category learning. Memory \& Cognition, 30(5), 666-677.

Ashby, F. G., Queller, S., \& Berrety, P. M. (1999). On the dominance of unidimensional rules in unsupervised classification. Perception \& Psychophysics, 61(6), 1178-1199.

Best, C. T., McRoberts, G. W., \& Sithole, N. M. (1988). Examination of perceptual reorganization for nonnative speech contrasts: Zulu click discrimination by English-speaking adults and infants. Journal of Experimental Psychology: Human Perception and Performance, 14(3), 345-360.

Bion, R. A. H., Miyazawa, K., Kikuchi, H., \& Mazuka, R. (2013). Learning Phonemic Vowel Length from Naturalistic Recordings of Japanese Infant-Directed Speech. PLoS ONE, 8(2), e51594. doi:10.1371/journal.pone.0051594

Bradlow, A. R., \& Bent, T. (2008). Perceptual adaptation to non-native speech. Cognition, 106(2), 707729. doi:10.1016/j.cognition.2007.04.005

Castro, L., Wasserman, E. A., \& Lauffer, M. (2018). Unsupervised learning of complex associations in an animal model. Cognition, 173, 28-33. doi:https://doi.org/10.1016/j.cognition.2017.12.015

Chandrasekaran, B., Koslov, S. R., \& Maddox, W. T. (2014). Toward a dual-learning systems model of speech category learning. Frontiers in Psychology, 5(825). doi:10.3389/fpsyg.2014.00825 
Chandrasekaran, B., Yi, H.-G., \& Maddox, W. T. (2014). Dual-learning systems during speech category learning. Psychonomic Bulletin \& Review, 21(2), 488-495. doi:10.3758/s13423-013-0501-5

Cole, J. S., Linebaugh, G., Munson, C., \& McMurray, B. (2010). Unmasking the acoustic effects of vowel-to-vowel coarticulation: A statistical modeling approach. Journal of Phonetics, 38(2), 167184.

Cooke, M., Barker, J., Lecumberri, M. L. G., \& Wasilewski, K. (2011). Crowdsourcing for word recognition in noise. Paper presented at the Twelfth Annual Conference of the International Speech Communication Association, Florence, Italy.

Cristia, A., \& Seidl, A. (2013). The hyperarticulation hypothesis of infant-directed speech. Journal of Child Language, FirstView, 1-22. doi:doi:10.1017/S0305000912000669

Davis, M. H., Johnsrude, I. S., Hervais-Adelman, A., Taylor, K., \& McGettigan, C. (2005). Lexical Information Drives Perceptual Learning of Distorted Speech: Evidence From the Comprehension of Noise-Vocoded Sentences. Journal of Experimental Psychology: General, 134(2), 222-241.

de Boer, B., \& Kuhl, P. K. (2003). Investigating the role of infant-directed speech with a computer model. Auditory Research Letters On-Line (ARLO), 4, 129-134.

Dich, N., \& Cohn, A. C. (2013). A review of spelling acquisition: Spelling development as a source of evidence for the psychological reality of the phoneme. Lingua, 133, 213-229.

Dietrich, C., Swingley, D., \& Werker, J. F. (2007). Native language governs interpretation of salient speech sound differences at 18 months. Proceedings of the National Academy of Sciences, 104(41), 16027-16031. doi:10.1073/pnas.0705270104

Earle, F. S., \& Myers, E. B. (2015). Sleep and native language interference affect non-native speech sound learning. Journal of Experimental Psychology: Human Perception and Performance, 41(6), 1680.

Eilers, R. E., Wilson, W., \& Moore, J. (1977). Developmental changes in speech discrimination in infants. Perception \& Psychophysics, 16, 513-521.

Eimas, P. D., Siqueland, E. R., Jusczyk, P., \& Vigorito, J. (1971). Speech perception in infants. Science(171), 303-306.

Eisner, F., McGettigan, C., Faulkner, A., Rosen, S., \& Scott, S. K. (2010). Inferior frontal gyrus activation predicts individual differences in perceptual learning of cochlear-implant simulations. The Journal of Neuroscience, 30(21), 7179-7186.

Escudero, P., Benders, T., \& Wanrooij, K. (2011). Enhanced bimodal distributions facilitate the learning of second language vowels. The Journal of the Acoustical Society of America, 130(4), EL206EL212. doi:10.1121/1.3629144

Escudero, P., \& Williams, D. (2014). Distributional learning has immediate and long-lasting effects. Cognition, 133(2), 408-413. doi:https://doi.org/10.1016/j.cognition.2014.07.002

Espy-Wilson, C. Y. (1992). Acoustic measures for linguistic features distinguishing the semivowels /w j r 1/ in American English. The Journal of the Acoustical Society of America, 92(2), 736-757. doi:10.1121/1.403998

Feldman, N. H., Griffiths, T. L., Goldwater, S., \& Morgan, J. L. (2013). A role for the developing lexicon in phonetic category acquisition. Psychological Review, 120(4), 751-778. doi:10.1037/a0034245

Francis, A. L., Ciocca, V., Ma, L., \& Fenn, K. (2008). Perceptual learning of Cantonese lexical tones by tone and non-tone language speakers. Journal of Phonetics, 36(2), 268-294. doi:https://doi.org/10.1016/j.wocn.2007.06.005

Francis, A. L., Kaganovich, N., \& Driscoll-Huber, C. (2008). Cue-specific effects of categorization training on the relative weighting of acoustic cues to consonant voicing in English. The Journal of the Acoustical Society of America, 124(2), 1234-1251. doi:10.1121/1.2945161

Freedberg, M., Glass, B., Filoteo, J. V., Hazeltine, E., \& Maddox, W. T. (2017). Comparing the effects of positive and negative feedback in information-integration category learning. Memory \& Cognition, 45(1), 12-25. doi:10.3758/s13421-016-0638-3 
Fuhrmeister, P., Schlemmer, B., \& Myers, E. B. (2020). Adults Show Initial Advantages Over Children in Learning Difficult Nonnative Speech Sounds. Journal of Speech, Language, and Hearing Research, 63(8), 2667-2679. doi:doi:10.1044/2020_JSLHR-19-00358

Galle, M. E., \& McMurray, B. (2014). The development of voicing categories: A meta-analysis of 40 years of infant research. Psychonomic Bulletin and Review, 21(4), 884-906.

Gauthier, B., Shi, R., \& Xu, Y. (2007). Learning phonetic categories by tracking movements. Cognition, 103(1), 80-106. doi:https://doi.org/10.1016/j.cognition.2006.03.002

Geller, J., Holmes, A., Schwalje, A., Berger, J., Gander, P., Choi, I., \& McMurray, B. (submitted). Validating the Iowa Test of Consonant Perception. Journal of the Acoustical Society of America.

Gerrits, E., \& Schouten, M. E. H. (2004). Categorical perception depends on the discrimination task. Perception \& Psychophysics, 66(3), 363-376.

Giguère, G., \& Love, B. C. (2013). Limits in decision making arise from limits in memory retrieval. Proceedings of the National Academy of Sciences, 110(19), 7613-7618. doi:10.1073/pnas.1219674110

Goudbeek, M., Cutler, A., \& Smits, R. (2008). Supervised and unsupervised learning of multidimensionally varying non-native speech categories. Speech Communication, 50(2), 109125.

Goudbeek, M., Swingley, D., \& Smits, R. (2009). Supervised and unsupervised learning of multidimensional acoustic categories. Journal of Experimental Psychology: Human Perception and Performance, 35(6), 1913-1933. doi:10.1037/a0015781

Guediche, S., Fiez, J. A., \& Holt, L. L. (2016). Adaptive plasticity in speech perception: Effects of external information and internal predictions. Journal of Experimental Psychology: Human Perception and Performance, 42(7), 1048-1059. doi:10.1037/xhp0000196

Guenther, F. H., \& Gjaja, M. (1996). The perceptual magnet effect as an emergent property of neural map formation. Journal of the Acoustical Society of America, 100, 1111-1112.

Gureckis, T. M., \& Love, B. C. (2004). Common Mechanisms in Infant and Adult Category Learning. Infancy, 5(2), 173-198. doi:10.1207/s15327078in0502_4

Hay, J. F., Graf Estes, K., Wang, T., \& Saffran, J. R. (2015). From Flexibility to Constraint: The Contrastive Use of Lexical Tone in Early Word Learning. Child Development, 86(1), 10-22. doi:10.1111/cdev.12269

Hazan, V., \& Barrett, S. (2000). The development of phonemic categorization in children aged 6-12. Journal of Phonetics, 28(4), 377-396. doi:http://dx.doi.org/10.1006/jpho.2000.0121

Hillenbrand, J. M., Getty, L., Clark, M. J., \& Wheeler, K. (1995). Acoustic Characteristics of American English vowels. Journal of the Acoustical Society of America, 97(5), 3099-3111.

Holt, L. L., \& Lotto, A. J. (2006). Cue weighting in auditory categorization: Implications for first and second language acquisition. The Journal of the Acoustical Society of America, 119(5), 30593071.

Holt, L. L., \& Lotto, A. J. (2008). Speech Perception Within an Auditory Cognitive Science Framework. Current Directions in Psychological Science, 17(1), 42-46. doi:10.1111/j.14678721.2008.00545.x

Idemaru, K., \& Holt, L. L. (2011). Word recognition reflects dimension-based statistical learning. Journal of Experimental Psychology: Human Perception and Performance, 37(6), 1939-1956. doi:10.1037/a0025641

Jongman, A., Wayland, R., \& Wong, S. (2000). Acoustic characteristics of English fricatives. Journal of the Acoustical Society of America, 106, 1252-1263.

Jusczyk, P. W. (1993). From general to language-specific capacities: The WRAPSA model of how speech perception develops. Journal of Phonetics.

Kraljic, T., Brennan, S. E., \& Samuel, A. G. (2008). Accommodating variation: Dialects, idiolects, and speech processing. Cognition, 107(1), 54-81. doi:http://dx.doi.org/10.1016/j.cognition.2007.07.013 
Kuhl, P. K. (1979). Speech perception in early infancy: Perceptual constancy for spectrally dissimilar vowel categories. Journal of the Acoustical Society of America, 66, 1668-1679.

Kuhl, P. K. (2004). Early language acquisition: cracking the speech code. Nat Rev Neurosci, 5(11), 831843.

Kuhl, P. K., Stevens, E. H., A., Deguchi, T., Kiritani, S., \& Iverson, P. (2006). Infants show a facilitation effect for native language phonetic perception between 6 and 12 months. Developmental Science, 9, F13-F21.

Lewandowsky, S., Yang, L.-X., Newell, B. R., \& Kalish, M. L. (2012). Working memory does not dissociate between different perceptual categorization tasks. Journal of Experimental Psychology: Learning, Memory, and Cognition, 38(4), 881-890.

Liberman, A. M., Harris, K. S., Hoffman, H. S., \& Griffith, B. C. (1957). The discrimination of speech sounds within and across phoneme boundaries. Journal of Experimental Psychology, 54(5), 358368.

Lieder, I., Adam, V., Frenkel, O., Jaffe-Dax, S., Sahani, M., \& Ahissar, M. (2019). Perceptual bias reveals slow-updating in autism and fast-forgetting in dyslexia. Nature Neuroscience, 22(2), 256-264. doi:10.1038/s41593-018-0308-9

Lim, S.-j., \& Holt, L. L. (2011). Learning foreign sounds in an alien world: Videogame training improves non-native speech categorization. Cognitive Science, 35(7), 1390-1405. doi:10.1111/j.15516709.2011.01192.x

Lisker, L. (1986). "Voicing" in English: a catalogue of acoustic features signaling /b/ versus /p/ in trochees. Language and Speech, 29(1), 3-11.

Liu, R., \& Holt, L. L. (2011). Neural Changes Associated with Nonspeech Auditory Category Learning Parallel Those of Speech Category Acquisition. Journal of Cognitive Neuroscience, 23(3), 683698. doi:10.1162/jocn.2009.21392

Lively, S. E., Logan, J. S., \& Pisoni, D. B. (1993). Training Japanese listeners to identify English/r/and/ 1/ II: The role of phonetic environment and talker variability in learning new perceptual categories. Journal of the Acoustical Society of America, 94, 1242-1255.

Logan, J. S., Lively, S. E., \& Pisoni, D. B. (1991). Training Japanese listeners to identify English/r/and/l: A first report. The Journal of the Acoustical Society of America, 89(2), 874-886.

Love, B. C., \& Gureckis, T. M. (2005). Modeling learning under the influence of culture. In W.-k. Ahn, R. L. Goldstone, B. C. Love, A. B. Markman, \& P. Wolff (Eds.), APA decade of behavior series. Categorization inside and outside the laboratory: Essays in honor of Douglas L. Medin (pp. 229248). Washington DC: American Psychological Association.

Love, B. C., Medin, D. L., \& Gureckis, T. M. (2004). SUSTAIN: a network model of category learning. Psychological Review, 111(2), 309.

Maddox, W. T., Ashby, F. G., \& Bohil, C. J. (2003). Delayed Feedback Effects on Rule-Based and Information-Integration Category Learning. Journal of Experimental Psychology: Learning, Memory \& Cognition, 29(4), 650-662.

Maddox, W. T., Chandrasekaran, B., Smayda, K., \& Yi, H.-G. (2013). Dual systems of speech category learning across the lifespan. Psychology and aging, 28(4), 1042-1056. doi:10.1037/a0034969

Maddox, W. T., \& Ing, A. D. (2005). Delayed Feedback Disrupts the Procedural-Learning System but Not the Hypothesis-Testing System in Perceptual Category Learning. Journal of Experimental Psychology: Learning, Memory, and Cognition, 31(1), 100-107. doi:10.1037/0278-7393.31.1.100

Martin, A., Schatz, T., Versteegh, M., Miyazawa, K., Mazuka, R., Dupoux, E., \& Cristia, A. (2015). Mothers speak less clearly to infants than to adults: A comprehensive test of the hyperarticulation hypothesis. Psychological Science, 26(3), 341-347.

Massaro, D. W., \& Cohen, M. M. (1983). Categorical or continuous speech perception: a new test. Speech Communication, 2, 15-35.

Maye, J., Aslin, R. N., \& Tanenhaus, M. K. (2008). The weckud wetch of the wast: Lexical adaptation to a novel accent. Cognitive Science: A Multidisciplinary Journal, 32(3), 543-562. doi:10.1080/03640210802035357 
Maye, J., \& Gerken, L. (2000). Learning phonemes without minimal pairs. Paper presented at the Proceedings of the 24th annual Boston university conference on language development.

Maye, J., Weiss, D. J., \& Aslin, R. N. (2008). Statistical phonetic learning in infants: Facilitation and feature generalization. Developmental Science, 11(1), 122-134. doi:10.1111/j.14677687.2007.00653.x

Maye, J., Werker, J. F., \& Gerken, L. (2003). Infant sensitivity to distributional information can affect phonetic discrimination. Cognition, 82, 101-111.

McCandliss, B. D., Fiez, J. A., Protopapas, A., Conway, M., \& McClelland, J. L. (2002). Success and failure in teaching the [r]-[1] contrast to Japanese adults: tests of a Hebbian model of plasticity and stabilization in spoken language perception. Cognitive, Affective, \& Behavioral Neuroscience, 2, 89-108.

McMurray, B., Aslin, R. N., \& Toscano, J. C. (2009). Statistical learning of phonetic categories: Insights from a computational approach. Developmental Science, 12(3), 369-379.

McMurray, B., Danelz, A., Rigler, H., \& Seedorff, M. (2018). Speech categorization develops slowly through adolescence. Developmental Psychology, 54(8), 1472-1491.

McMurray, B., \& Jongman, A. (2011). What information is necessary for speech categorization? Harnessing variability in the speech signal by integrating cues computed relative to expectations. Psychological Review, 118(2), 219-246.

McMurray, B., Kovack-Lesh, K., Goodwin, D., \& McEchron, W. D. (2013). Infant directed speech and the development of speech perception: Enhancing development or an unintended consequence? . Cognition, 129, 362-378.

Metsala, J. L., \& Walley, A. C. (1998). Spoken vocabulary growth and the segmental restructuring of lexical representations: Precursors to phonemic awareness and early reading ability. In J. L. Metsala \& L. Ehri (Eds.), Word recognition in beginning literacy (pp. 89-120). Mahwah, NJ: Lawrence Erlbaum Associates.

Mirman, D., Holt, L. L., \& McClelland, J. L. (2004). Categorization and discrimination of nonspeech sounds: Differences between steady-state and rapidly-changing acoustic cues. The Journal of the Acoustical Society of America, 116(2), 1198-1207. doi:10.1121/1.1766020

Mirman, D., Magnuson, J. S., Estes, K. G., \& Dixon, J. A. (2008). The link between statistical segmentation and word learning in adults. Cognition, 108(1), 271-280.

Miyazawa, K., Shinya, T., Martin, A., Kikuchi, H., \& Mazuka, R. (2017). Vowels in infant-directed speech: More breathy and more variable, but not clearer. Cognition, 166, 84-93.

Narayan, C. R., Werker, J. F., \& Beddor, P. S. (2010). The interaction between acoustic salience and language experience in developmental speech perception: evidence from nasal place discrimination. Developmental Science, 13(3), 407-420. doi:10.1111/j.1467-7687.2009.00898.x

Nittrouer, S. (2004). The role of temporal and dynamic signal components in the perception of syllablefinal stop voicing by children and adults. The Journal of the Acoustical Society of America, 115(4), 1777-1790. doi:doi:http://dx.doi.org/10.1121/1.1651192

Nittrouer, S., \& Miller, M. E. (1997). Predicting developmental shifts in perceptual weighting schemes. Journal of the Acoustical Society of America, 101, 2253-2266.

Norris, D., McQueen, J., \& Cutler, A. (2003). Perceptual learning in speech. Cognitive Psychology, 47(2), 204-238.

Ohde, R. N. (1984). Fundamental frequency as an acoustic correlate of stop consonant voicing. Journal of the Acoustical Society of America, 75(1), 224-230.

Perrachione, T. K., Lee, J., Ha, L., \& Wong, P. C. M. (2011). Learning a novel phonological contrast depends on interactions between individual differences and training paradigm design. . Journal of the Acoustical Society of America, 130 (1), 461-472.

Peterson, G. E., \& Barney, H. L. (1952). Control methods used in a study of the vowels. Journal of the Acoustical Society of America, 24, 175-184. 
Pisoni, D. B., Aslin, R. N., Perey, A. J., \& Hennessy, B. L. (1982). Some effects of laboratory training on identification and discrimination of voicing contrasts in stop consonants. Journal of Experimental Psychology: Human Perception and Performance, 8(2), 297-314. doi:10.1037/0096-1523.8.2.297

Reetzke, R., Maddox, W. T., \& Chandrasekaran, B. (2016). The Role of Age and Executive Function in Auditory Category Learning. Journal of Experimental Child Psychology, 142, 48-65. doi:10.1016/j.jecp.2015.09.018

Richler, J. J., \& Palmeri, T. J. (2014). Visual category learning. Wiley Interdisciplinary Reviews: Cognitive Science, 5(1), 75-94.

Roark, C. L., \& Holt, L. L. (2018). Task and distribution sampling affect auditory category learning. Attention, Perception, \& Psychophysics, 80(7), 1804-1822. doi:10.3758/s13414-018-1552-5

Rost, G. C., \& McMurray, B. (2009). Speaker variability augments phonological processing in early word learning. Developmental Science, 12(2), 339-349.

Rost, G. C., \& McMurray, B. (2010). Finding the signal by adding noise: The role of non-contrastive phonetic variability in early word learning. Infancy, 15(6), 608.

Samuel, A. G., \& Kraljic, T. (2009). Perceptual learning for speech. Attention, Perception, \& Psychophysics, 71(6), 1207-1218. doi:10.3758/app.71.6.1207

Schouten, M. E. H., Gerrits, E., \& Van Hessen, A. (2003). The end of categorical perception as we know it. Speech Communication, 41, 71-80.

Seedorff, M., Oleson, J., \& McMurray, B. (submitted). Maybe maximal: Good enough mixed models optimize power while controlling type I error. https://psyarxiv.com/xmhfr/.

Slawinski, E. B., \& Fitzgerald, L. K. (1998). Perceptual development of the categorization of the/r-w/ contrast in normal children. Journal of Phonetics, 26, 27-43.

Slote, J., \& Strand, J. F. (2016). Conducting spoken word recognition research online: Validation and a new timing method. Behavioral Research Methods, 48(2), 553-566.

Smits, R., Sereno, J., \& Jongman, A. (2006). Categorization of sounds. Journal of Experimental Psychology: Human Perception and Performance, 32(3), 733-754.

Strange, W., \& Shafer, V. L. (2008). Speech perception in second langauge learners: The re-education of selective perception. In J. G. H. Hansen \& M. Zampini (Eds.), Phonology and Second Language Acquisition (pp. 153-192). Philadelphia: John Benjamins.

Summerfield, Q., \& Haggard, M. (1977). On the dissociation of spectral and temporal cues to the voicing distinction in initial stop consonants. Journal of the Acoustical Society of America, 62(2), 435448.

Thiessen, E. D. (2007). The effect of distributional information on children's use of phonemic contrasts. Journal of Memory and Language, 56(1), 16-34.

Toscano, J. C., \& McMurray, B. (2010). Cue integration with categories: Weighting acoustic cues in speech using unsupervised learning and distributional statistics. Cognitive Science, 34(3), 436464.

Toscano, J. C., McMurray, B., Dennhardt, J., \& Luck, S. (2010). Continuous Perception and Graded Categorization Electrophysiological Evidence for a Linear Relationship Between the Acoustic Signal and Perceptual Encoding of Speech. Psychological Science, 21(10), 1532-1540.

Tsuji, S., \& Cristia, A. (2014). Perceptual attunement in vowels: A meta-analysis. Developmental psychobiology, 56(179-191).

Vallabha, G. K., McClelland, J. L., Pons, F., Werker, J. F., \& Amano, S. (2007). Unsupervised learning of vowel categories from infant-directed speech. . Proceedings of the National Academy of Sciences, 104, 13273-13278.

Wade, T., \& Holt, L. L. (2005). Incidental categorization of spectrally complex non-invariant auditory stimuli in a computer game task. The Journal of the Acoustical Society of America, 118(4), 26182633.

Walley, A. C., Metsala, J. L., \& Garlock, V. M. (2003). Spoken vocabulary growth: Its role in the development of phoneme awareness and early reading ability. Reading and Writing, 16(1), 5-20. 
Werker, J. F., \& Curtin, S. (2005). PRIMIR: A Developmental Framework of Infant Speech Processing. Language Learning and Development, 1(2), 197-234. doi:10.1080/15475441.2005.9684216

Werker, J. F., \& Tees, R. C. (1984). Cross-language speech perception: Evidence for perceptual reorganization during the first year of life. Infant Behavior and Development, 7, 49-63.

Westermann, G., \& Miranda, E. R. (2004). A new model of sensorimotor coupling in the development of speech. Brain and Language, 89(2), 393-400.

Wong, P. C. M., Perrachione, T. K., \& Parrish, T. B. (2007). Neural characteristics of successful and less successful speech and word learning in adults. Human Brain Mapping, 28(10), 995-1006. doi:doi:10.1002/hbm.20330

Wong, P. C. M., Warrier, C. M., Penhune, V. B., Roy, A. K., Sadehh, A., Parrish, T. B., \& Zatorre, R. J. (2008). Volume of Left Heschl's Gyrus and Linguistic Pitch Learning. Cerebral Cortex, 18(4), 828-836. doi:10.1093/cercor/bhm115

Woods, K. J. P., Siegel, M. H., Traer, J., \& McDermott, J. H. (2017). Headphone screening to facilitate web-based auditory experiments. Attention, Perception, and Psychophysics, 79(7), 2064-2072. doi:10.3758/s13414-017-1361-2

Yeung, H. H., \& Werker, J. F. (2009). Learning words' sounds before learning how words sound: 9month-olds use distinct objects as cues to categorize speech information. Cognition, 113(2), 234243.

Yi, H.-G., \& Chandrasekaran, B. (2016). Auditory categories with separable decision boundaries are learned faster with full feedback than with minimal feedback. The Journal of the Acoustical Society of America, 140(2), 1332-1335. doi:10.1121/1.4961163

Yi, H.-G., Maddox, W. T., Mumford, J. A., \& Chandrasekaran, B. (2016). The Role of Corticostriatal Systems in Speech Category Learning. Cerebral Cortex, 26(4), 1409-1420.

doi:10.1093/cercor/bhu236 


\title{
Reinforced statistical learning of auditory categories \\ ONLINE SUPPLEMENT
}

\author{
Bob McMurray \\ and \\ Samantha L. Chiu \\ Dept. of Psychological and Brain Sciences \\ University of Iowa
}




\section{S1. Complete Results of LME models for training data.}

This model examined accuracy on training trials for all subjects. Its structure is described by 1 .

$$
\begin{aligned}
\text { Correct } \sim & \left(\text { trial }+\operatorname{trial}^{2}+\operatorname{trial}^{3}\right) * \text { UnsupMatch } *(\mathrm{RB}+\mathrm{wII}+\mathrm{wRB})+ \\
& \left(\text { trial }+\operatorname{trial}^{2}+\operatorname{trial}^{3} \| \text { Subject }\right)
\end{aligned}
$$

UnsupMatch: $\quad$ whether the supervised boundary matched the unsupervised;

RB: $\quad$ supervised boundary was vertical or horizontal boundary vs. diagonal;

wRB (within RB): contrast between horizontal and vertical supervised boundary

wII (within II): $\quad$ contrast between diagonal-left and diagonal-right supervised boundary.

\begin{tabular}{|c|c|c|c|c|c|c|}
\hline & Fixed Effect & B & SE & $\mathbf{Z}$ & $\mathbf{p}$ & sig \\
\hline \multirow{4}{*}{ 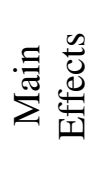 } & (Intercept) & 1.46 & 0.06 & 25.07 & $<0.0001$ & $* * *$ \\
\hline & trial & -0.10 & 0.10 & -1.01 & 0.31 & \\
\hline & trial $^{2}$ & -0.39 & 0.17 & -2.24 & 0.0250 & * \\
\hline & trial $^{3}$ & 3.10 & 0.53 & 5.83 & $<0.0001$ & $* * *$ \\
\hline \multirow{3}{*}{ 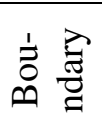 } & $\mathrm{RB}$ & 0.13 & 0.12 & 1.16 & 0.25 & \\
\hline & wII & 0.54 & 0.16 & 3.29 & 0.00101 & $* *$ \\
\hline & wRB & -0.25 & 0.16 & -1.53 & 0.13 & \\
\hline \multirow{9}{*}{ 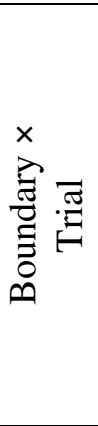 } & $\mathrm{RB} \times$ trial & 0.04 & 0.20 & 0.22 & 0.82 & \\
\hline & $\times$ trial $^{2}$ & 0.51 & 0.34 & 1.49 & 0.14 & \\
\hline & $\times$ trial $^{3}$ & -1.14 & 1.07 & -1.07 & 0.29 & \\
\hline & $\mathrm{wRB} \times$ trial & -0.02 & 0.28 & -0.08 & 0.94 & \\
\hline & $\times$ trial $^{2}$ & 0.83 & 0.49 & 1.68 & 0.0938 & + \\
\hline & $\times$ trial $^{3}$ & -0.45 & 1.56 & -0.29 & 0.77 & \\
\hline & wII $\times$ trial & -0.27 & 0.27 & -1.00 & 0.32 & \\
\hline & $\times \operatorname{trial}^{2}$ & -0.23 & 0.48 & -0.49 & 0.62 & \\
\hline & $\times$ trial $^{3}$ & 1.13 & 1.48 & 0.76 & 0.45 & \\
\hline \multirow{4}{*}{ 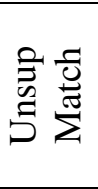 } & UnsupMatch & 0.04 & 0.12 & 0.37 & 0.71 & \\
\hline & UnsupMatch $\times$ trial & 0.00 & 0.20 & -0.01 & 0.99 & \\
\hline & $\times$ trial $^{2}$ & 0.03 & 0.34 & 0.10 & 0.92 & \\
\hline & $\times$ trial $^{3}$ & 0.37 & 1.07 & 0.34 & 0.73 & \\
\hline \multirow{12}{*}{ 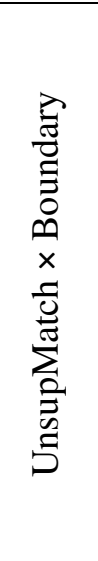 } & UnsupMatch $\times \mathrm{RB}$ & -0.18 & 0.23 & -0.77 & 0.44 & \\
\hline & $\times w R B$ & -0.01 & 0.33 & -0.03 & 0.97 & \\
\hline & $\times$ wII & 0.32 & 0.33 & 0.97 & 0.33 & \\
\hline & UnsupMatch $\times \mathrm{RB} \times$ trial & 0.14 & 0.39 & 0.36 & 0.72 & \\
\hline & $\times$ trial $^{2}$ & 1.43 & 0.69 & 2.08 & 0.0373 & $*$ \\
\hline & $\times \operatorname{trial}^{3}$ & -0.29 & 2.15 & -0.14 & 0.89 & \\
\hline & UnsupMatch $\times$ wRB $\times$ trial & 0.47 & 0.57 & 0.83 & 0.41 & \\
\hline & $\times$ trial $^{2}$ & 0.63 & 1.00 & 0.63 & 0.53 & \\
\hline & $\times$ trial $^{3}$ & -1.60 & 3.13 & -0.51 & 0.61 & \\
\hline & UnsupMatch $\times$ wII $\times$ trial & -1.21 & 0.54 & -2.25 & 0.0248 & $*$ \\
\hline & $\times$ trial $^{2}$ & -0.26 & 0.95 & -0.27 & 0.70 & \\
\hline & $\times$ trial $^{3}$ & 6.74 & 2.92 & 2.31 & 0.0210 & $*$ \\
\hline
\end{tabular}

Table S1.1: Results of a logistic mixed effects modeling examining all training trials and subject.

$+\mathrm{p}<.1 ; * \mathrm{p}<.05, * * \mathrm{p}<.01, * * * \mathrm{p}<.001$ 
The same model was also applied to the training trials for the restricted set of subjects. Model formula and variables are identical to $\mathrm{S} 1$.

Table S1.2: Results of a logistic mixed effects modeling examining all training trials and the more restricted set of subjects. Sig (All) column replicates significance column from prior analysis (Table S1.1)

\begin{tabular}{|c|c|c|c|c|c|c|c|}
\hline & Fixed Effect & B & SE & $\mathbf{Z}$ & $\mathbf{p}$ & sig & sig (all) \\
\hline \multirow{4}{*}{ 怘 } & (Intercept) & 1.57 & 0.06 & 28.25 & $<0.0001$ & $* * *$ & $* * *$ \\
\hline & trial & 0.00 & 0.11 & 0.03 & 0.98 & & \\
\hline & trial $^{2}$ & -0.57 & 0.20 & -2.90 & 0.00374 & $* *$ & $*$ \\
\hline & trial $^{3}$ & 2.97 & 0.59 & 4.99 & $<0.0001$ & $* * *$ & $* * *$ \\
\hline \multirow{3}{*}{ 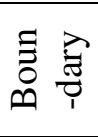 } & $\mathrm{RB}$ & 0.20 & 0.11 & 1.77 & 0.0760 & + & \\
\hline & wII & 0.47 & 0.16 & 2.93 & 0.00344 & $* *$ & $* *$ \\
\hline & wRB & -0.39 & 0.15 & -2.53 & 0.0114 & $*$ & \\
\hline \multirow{9}{*}{ 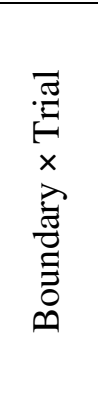 } & $\mathrm{RB} \times$ trial & 0.01 & 0.22 & 0.03 & 0.97 & & \\
\hline & $\times$ trial $^{2}$ & 0.56 & 0.39 & 1.43 & 0.15 & & \\
\hline & $\times$ trial $^{3}$ & -0.86 & 1.19 & -0.73 & 0.47 & & \\
\hline & $\mathrm{wRB} \times$ trial & 0.01 & 0.30 & 0.03 & 0.97 & & \\
\hline & $\times$ trial $^{2}$ & 1.08 & 0.56 & 1.95 & 0.0511 & + & + \\
\hline & $\times$ trial $^{3}$ & -0.92 & 1.69 & -0.54 & 0.59 & & \\
\hline & wII $\times$ trial & -0.48 & 0.31 & -1.56 & 0.118 & & \\
\hline & $\times$ trial $^{2}$ & -0.22 & 0.56 & -0.40 & 0.69 & & \\
\hline & $\times$ trial $^{3}$ & 1.70 & 1.67 & 1.02 & 0.31 & & \\
\hline \multirow{4}{*}{ 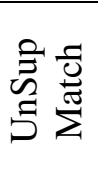 } & UnsupMatch & 0.04 & 0.11 & 0.38 & 0.70 & & \\
\hline & UnsupMatch $\times$ trial & 0.04 & 0.22 & 0.18 & 0.86 & & \\
\hline & $\times$ trial $^{2}$ & -0.14 & 0.39 & -0.36 & 0.72 & & \\
\hline & $\times$ trial $^{3}$ & 0.11 & 1.19 & 0.09 & 0.93 & & \\
\hline \multirow{12}{*}{ 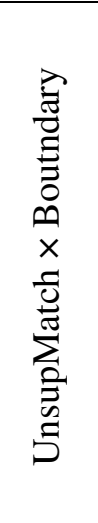 } & UnsupMatch $\times$ RB & -0.38 & 0.22 & -1.71 & 0.0873 & + & \\
\hline & $\times w R B$ & 0.02 & 0.31 & 0.08 & 0.94 & & \\
\hline & $\times$ wII & 0.07 & 0.32 & 0.23 & 0.82 & & \\
\hline & UnsupMatch $\times \mathrm{RB} \times$ trial & 0.17 & 0.43 & 0.40 & 0.69 & & \\
\hline & $\times$ trial $^{2}$ & 1.65 & 0.79 & 2.10 & 0.0362 & * & * \\
\hline & $\times$ trial $^{3}$ & -1.00 & 2.38 & -0.42 & 0.68 & & \\
\hline & UnsupMatch $\times w R B \times$ trial & 0.17 & 0.61 & 0.28 & 0.78 & & \\
\hline & $\times$ trial $^{2}$ & 0.45 & 1.11 & 0.41 & 0.68 & & \\
\hline & $\times$ trial $^{3}$ & -0.34 & 3.38 & -0.10 & 0.92 & & \\
\hline & UnsupMatch $\times$ wII $\times$ trial & -1.06 & 0.61 & -1.73 & 0.0839 & + & $*$ \\
\hline & $\times$ trial $^{2}$ & -0.05 & 1.12 & -0.05 & 0.96 & & \\
\hline & $\times$ trial $^{3}$ & 5.19 & 3.35 & 1.55 & 0.121 & & $*$ \\
\hline
\end{tabular}

$+\mathrm{p}<.1 ; * \mathrm{p}<.05, * * \mathrm{p}<.01, * * * \mathrm{p}<.001$ 


\section{S2. Analysis of Tone 1 / Tone 2 as an integral dimension.}

The structure of the categories we tested here was motivated by the RB/II distinction claimed by COVIS (Ashby \& Maddox, 2011) and its recent extensions to auditory category learning (Chandrasekaran, Yi, \& Maddox, 2014; Maddox, Chandrasekaran, Smayda, \& Yi, 2013; Roark \& Holt, 2018; Yi \& Chandrasekaran, 2016). The categories were meant to simulate something like vowels where the frequency of each band of energy serves as an independent feature.

However, the results of the training trials suggested that this characterization of the cue-space did not predict performance. The diagonal-left category boundary (an II category) appeared to be learned the best, followed by the vertical, then horizontal (both RB), and then the diagonal-right (see Main Text, Figures 3 and 7A). One explanation for this pattern is that the Tone 1 and Tone 2 frequencies were not treated as independent cues (they were at least partially integral cues). For example, listeners could compute a combined pitch cue and attempt to locate the categories along this single dimension. This integral cue could be potential used along side the other two cues.

The consequences of this was particularly apparent when working through the results of the rotation procedure for the two diagonal categories (Figure S2.1). In panel A we see the diagonal-left categories plotted in Bark space, along with the coordinates of a grid of Tone $1 \times$ Tone 2 frequencies surrounding the categories. In panel B both the categories and the grid points have been rotated such that the $\mathrm{X}$ axis is now relevant, and the $\mathrm{Y}$ axis is irrelevant. Here, reading the original grid points horizontally (along
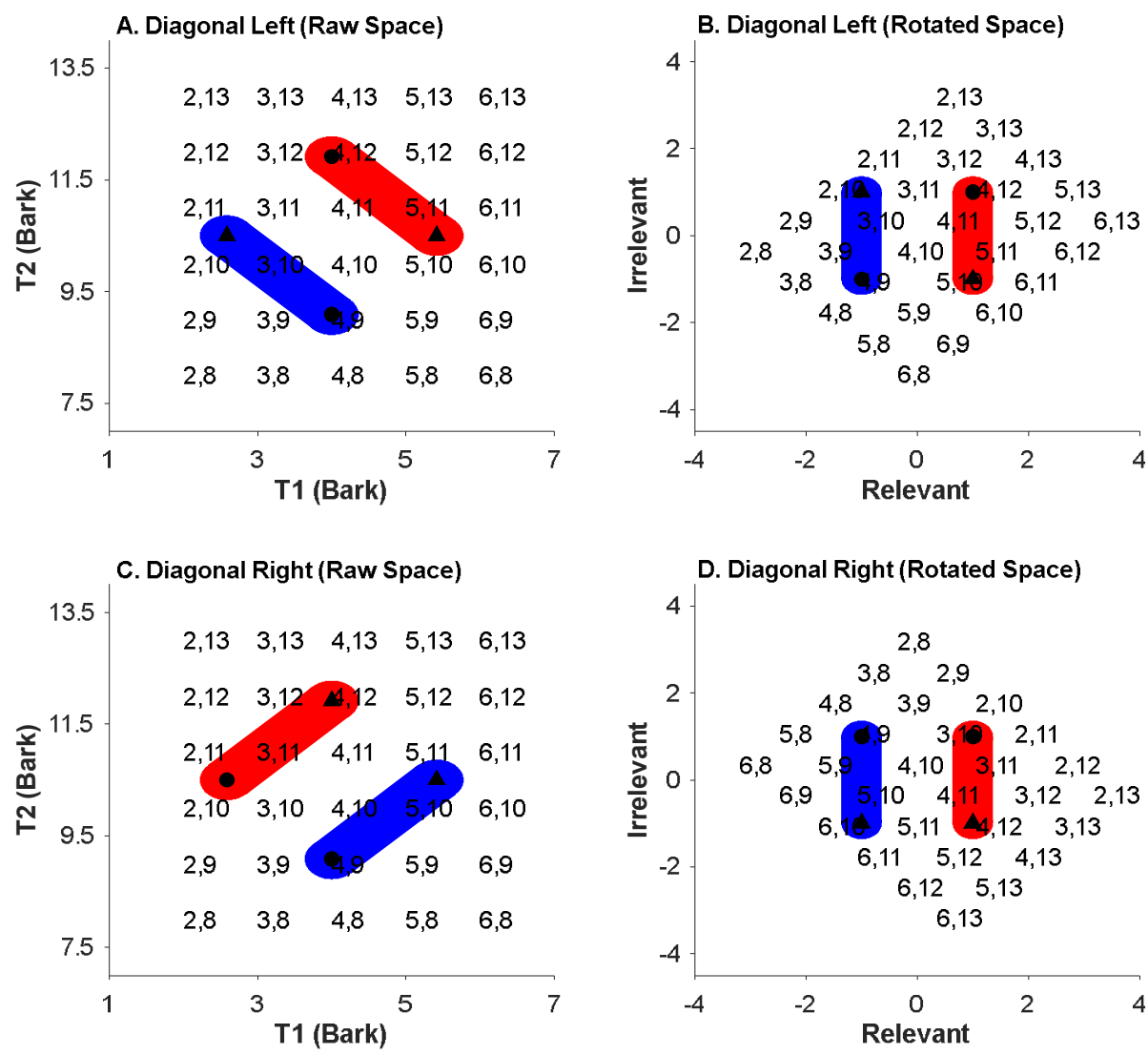

Figure S2.1. A) The diagonal-left categories in raw frequency space. The blue category is category A. Grid points labels are plotted in their approximate location. B) Grid points and categories in rotated space. The labels of the gridpoints reflect their original (Tone $1 \times$ Tone 2) location. C) Diagonal-right category in raw space. D) Diagonal-right category in rotated space. 

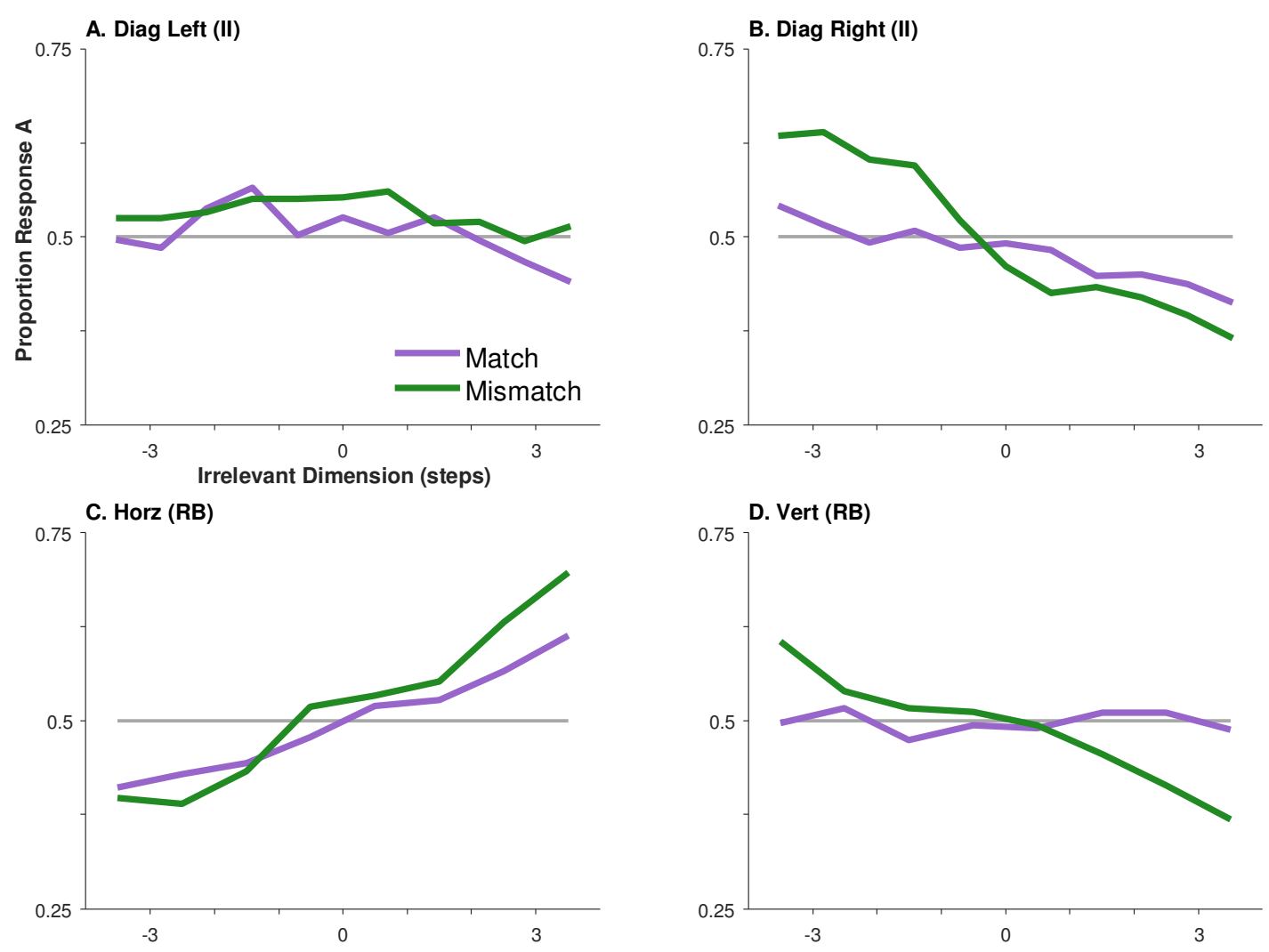

Figure S2.2: Proportion response A as a function of the irrelevant dimension (after rotation), and the supervised/unsupervised match for all subjects. A) For the diagonal-left supervised boundary; B) for the diagonal-right boundary; C) for the horizontal boundary; D) for the vertical boundary.

the relevant dimension), we see that both frequencies increase as the relevant dimension increases.

Thus, in this case, if the listeners are already computing an integral pitch cue, they already have the right cue weighting. They should be able to learn these categories rapidly and show little use of the irrelevant dimension. This is exactly what was observed in the empirical data (Figure S2.2A, a duplicate of Figure 8 in the main text).

In contrast, for the diagonal-right categories (Figure S2.1C, D) we see the opposite. After rotation, the two original tone frequencies work in opposition: as the relevant dimension goes from low to high, Tone 1 decreases its frequency, and Tone 2 increases it. If listeners are using an average of the two cues, then this predicts that listeners should struggle to learn these categories. Generally speaking, higher values of Tone 1 are associated with Category A (the blue category is to the right of the red in panel C). If listeners were also using Tone 1 (along with the integral cue) this predicts that at lower values of the irrelevant dimension (high Tone 1), we should see more Category A responses and this should fall as the irrelevant dimension increases-exactly what is observed in Figure S2.2B).

Figure S2.3A shows the same for the vertical categories. Here, even though Tone 2 is irrelevant, it contributes to the overall pitch percept, and can thus bias Tone 1. Normally greater Tone 1 pitch is associated with fewer Category A (blue) responses. Consequently, when the irrelevant dimension (Tone 2) increases, the rate of Category A responses should decrease. This is what was observed in the empirical results (see Figure S2.2D), particularly when the unsupervised learning did not match. 

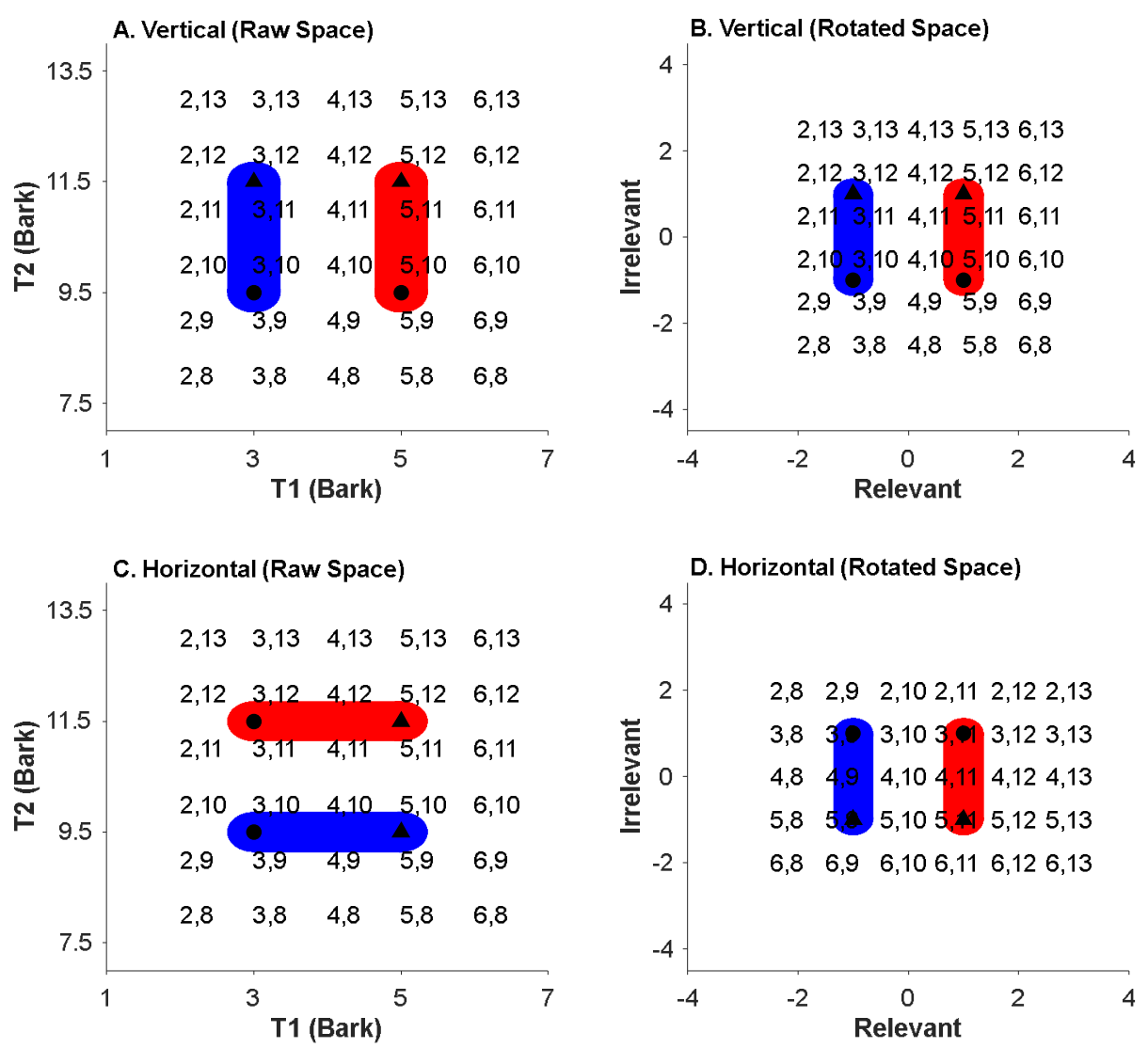

Figure S2.3. C) The vertical categories in raw frequency space. The blue category is category A. Grid points labels are plotted in their approximate location. B) Grid points and categories in rotated space. The labels of the gridpoints reflect their original (Tone $1 \times$ Tone 2) location. C) Horizontal categories in raw space. D) Horizontal category in rotated space.

Finally, Figure S2.3C and D show the same for the horizontal categories. In this case, after rotation, now Tone 2 is relevant and Tone 1 is not. Critically, however, if the Tone 1 frequency also contributes to Tone $2 \mathrm{~s}$ percept, then we predict the following pattern. As the irrelevant dimension increases (Panel D, Y axis), Tone 1 frequency decreases. Since lower frequencies are associated with more Category A responses, this predicts increasing category A responses as the irrelevant dimension increases. Again, this is exactly what was seen in the empirical data (Figure 8C, main text), particularly when the unsupervised distribution did not match.

Conclusion. This analysis suggests that if we assume that Tone 1 and Tone 2 frequencies are partially integral, then it makes two predictions that are borne out by the data. First, the diagonal-left categories should be the easiest to learn (as they capitalize on this assumed correlation), the diagonal-right categories should be the hardest and the vertical and horizontal should be in between. This is what was observed empirically, in both the training data (Figure 3, main text) and in the test trials in the pattern slope of the curve as a function of the relevant dimension (Figure 7A). Second, for all four categorytypes there are highly specific predictions as to the direction of the effect of the irrelevant dimension (after rotation). In all four cases, these predictions were borne out by the empirical data, particularly when the unsupervised learning did not match the supervised. 


\section{S3. Complete Results of LME models for test data.}

These models examined the testing trials. These predicted a category A response as a function of the stimulus properties after the Tone $1 \times$ Tone 2 space had been rotated into a common space, and after the new irrelevant dimension had been flipped (see Note 2). Its structure is described by 2 .

$$
\begin{aligned}
\text { Response } \sim & (\mathrm{RB}+\mathrm{wII}+\mathrm{wRB}) * \text { UnsupMatch }^{*} \mathrm{Rel} *\left(\text { Irrel }+ \text { Irrel }^{2}\right)+\text { LastResp*LastDis }+ \\
& \left(\text { Rel } *\left(\text { Irrel }+ \text { Irrel }^{2}\right)+\text { LastCat }^{*} \text { LastDist II Subject }\right)
\end{aligned}
$$

UnsupMatch: $\quad$ whether the supervised boundary matched the unsupervised;

RB: wRB (within RB): wII (within II): supervised boundary was vertical or horizontal boundary vs. diagonal;

Rel: contrast between horizontal and vertical supervised boundary

Irrel:

Irrel $^{2}$ : contrast between diagonal-left and diagonal-right supervised boundary. Relevant Dimension after rotation (based on step number of Tone 1/2) Irrelevant Dimension after rotation, and flipping to account for integral cues (based on step number of Tone 1/2)

LastResp: Quadratic term for Irrelevant Dimension

LastDist: Response on last trial (coded same as Response) Euclidean Distance between current stimulus and prior stimulus 
Table S3.1: Results of a logistic mixed effects model examining likelihood of responding ' $\mathrm{A}$ ' during testing trials for all subjects.

\begin{tabular}{|c|c|c|c|c|c|c|}
\hline & Fixed effect & B & SE & $\mathbf{Z}$ & $\mathbf{p}$ & sig \\
\hline \multirow{7}{*}{ 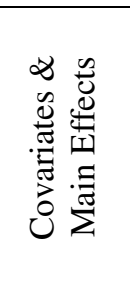 } & (Intercept) & 0.03 & 0.05 & 0.63 & 0.53 & \\
\hline & LastCat & 0.06 & 0.07 & 0.80 & 0.42 & \\
\hline & LastDist & -0.12 & 0.08 & -1.45 & 0.15 & \\
\hline & LastCat $\times$ LastDist & -2.92 & 0.22 & -13.23 & $<0.0001$ & $* * *$ \\
\hline & $\mathrm{RB}$ & -0.04 & 0.10 & -0.36 & 0.72 & \\
\hline & winII & 0.28 & 0.15 & 1.88 & 0.060 & + \\
\hline & winRB & 0.14 & 0.15 & 0.93 & 0.35 & \\
\hline \multirow{4}{*}{ 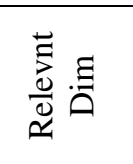 } & Rel & -6.43 & 0.31 & -20.42 & $<0.0001$ & $* * *$ \\
\hline & $\times \mathrm{RB}$ & -0.81 & 0.62 & -1.30 & 0.19 & \\
\hline & $\times$ wII & -2.72 & 0.88 & -3.10 & 0.002 & $* *$ \\
\hline & $x w R B$ & 1.90 & 0.89 & 2.14 & 0.032 & $*$ \\
\hline \multirow{8}{*}{ 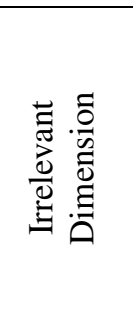 } & Irrel & -1.01 & 0.30 & -3.33 & 0.0009 & $* * *$ \\
\hline & $\times \mathrm{RB}$ & -0.45 & 0.60 & -0.75 & 0.45 & \\
\hline & $\times$ wII & 0.87 & 0.85 & 1.02 & 0.31 & \\
\hline & $x w R B$ & -0.74 & 0.86 & -0.86 & 0.39 & \\
\hline & Irrel $^{2}$ & -0.28 & 0.28 & -1.00 & 0.32 & \\
\hline & $\times \mathrm{RB}$ & 1.38 & 0.55 & 2.51 & 0.012 & $*$ \\
\hline & $\times$ wII & -1.76 & 0.79 & -2.24 & 0.025 & $*$ \\
\hline & $\times w R B$ & 0.74 & 0.77 & 0.97 & 0.33 & \\
\hline \multirow{8}{*}{ 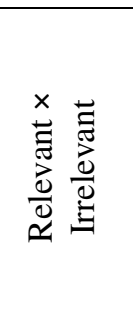 } & Rel $\times$ Irrel & -0.49 & 0.38 & -1.28 & 0.20 & \\
\hline & $\times \mathrm{RB}$ & 2.67 & 0.77 & 3.47 & 0.0005 & $* * *$ \\
\hline & $\times$ wII & -3.41 & 1.06 & -3.21 & 0.001 & $* *$ \\
\hline & $\times w R B$ & 0.82 & 1.11 & 0.74 & 0.46 & \\
\hline & Rel $\times$ Irrel $^{2}$ & 0.29 & 1.14 & 0.26 & 0.80 & \\
\hline & $\times \mathrm{RB}$ & 5.28 & 2.22 & 2.38 & 0.017 & $*$ \\
\hline & $\times$ wII & -5.73 & 3.04 & -1.89 & 0.059 & + \\
\hline & $\times w R B$ & 0.07 & 3.19 & 0.02 & 0.98 & \\
\hline \multirow{4}{*}{ 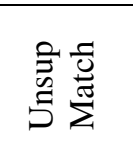 } & UnsupMatch & -0.13 & 0.10 & -1.27 & 0.21 & \\
\hline & $\times \mathrm{RB}$ & 0.07 & 0.21 & 0.35 & 0.72 & \\
\hline & $\times$ wII & -0.12 & 0.29 & -0.39 & 0.69 & \\
\hline & $\times w R B$ & -0.20 & 0.29 & -0.67 & 0.50 & \\
\hline \multirow{4}{*}{ 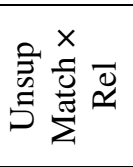 } & UnsupMatch $\times$ Rel & -0.07 & 0.62 & -0.11 & 0.91 & \\
\hline & $\times \mathrm{RB}$ & -0.03 & 1.26 & -0.02 & 0.98 & \\
\hline & $\times$ wII & -2.02 & 1.75 & -1.15 & 0.25 & \\
\hline & $\times w R B$ & 0.01 & 1.81 & 0.01 & 1.00 & \\
\hline \multirow{8}{*}{ 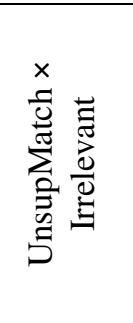 } & UnsupMatch $\times$ Irrel & 0.89 & 0.60 & 1.48 & 0.14 & \\
\hline & $\times \mathrm{RB}$ & 0.54 & 1.21 & 0.44 & 0.66 & \\
\hline & $\times$ wII & -1.90 & 1.70 & -1.12 & 0.26 & \\
\hline & $\times w R B$ & -0.63 & 1.72 & -0.37 & 0.71 & \\
\hline & UnsupMatch $\times$ Irrel $^{2}$ & -0.80 & 0.55 & -1.46 & 0.14 & \\
\hline & $\times \mathrm{RB}$ & 1.49 & 1.10 & 1.35 & 0.18 & \\
\hline & $\times$ wII & 0.48 & 1.59 & 0.30 & 0.76 & \\
\hline & $\times w R B$ & -1.33 & 1.53 & -0.87 & 0.38 & \\
\hline \multirow{8}{*}{ 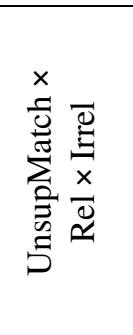 } & UnsupMatch $\times$ Rel $\times$ Irrel & -1.02 & 0.77 & -1.33 & 0.18 & \\
\hline & $\times \mathrm{RB}$ & -1.69 & 1.55 & -1.09 & 0.27 & \\
\hline & $\times$ wII & 0.55 & 2.15 & 0.26 & 0.80 & \\
\hline & $\times w R B$ & -1.71 & 2.22 & -0.77 & 0.44 & \\
\hline & UnsupMatch $\times$ Rel $\times$ Irrel $^{2}$ & 2.06 & 2.20 & 0.94 & 0.35 & \\
\hline & $\times \mathrm{RB}$ & -7.90 & 3.97 & -1.99 & 0.047 & $*$ \\
\hline & $\times$ wII & 0.63 & 5.96 & 0.11 & 0.92 & \\
\hline & $x w R B$ & 10.65 & 5.30 & 2.01 & 0.044 & $*$ \\
\hline
\end{tabular}

$+\mathrm{p}<.1 ; * \mathrm{p}<.05, * * \mathrm{p}<.01, * * * \mathrm{p}<.001$ 
Table S3.2: Results of a logistic mixed effects model examining likelihood of responding 'A' during testing trials for restricted set of subjects. Sig (All) column replicates significance column from prior analysis (Table S1.1)

\begin{tabular}{|c|c|c|c|c|c|c|c|}
\hline & Fixed effect & B & SE & $\mathbf{Z}$ & $\mathbf{p}$ & sig & sig (all) \\
\hline \multirow{7}{*}{ 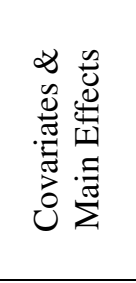 } & (Intercept) & 0.04 & 0.05 & 0.72 & 0.47 & & \\
\hline & LastCat & 0.10 & 0.07 & 1.41 & 0.16 & & \\
\hline & LastDist & -0.17 & 0.10 & -1.72 & 0.085 & + & \\
\hline & LastCat $\times$ LastDist & -3.00 & 0.25 & -12.17 & $<0.0001$ & $* * *$ & $* * *$ \\
\hline & RB & -0.06 & 0.10 & -0.55 & 0.58 & & \\
\hline & winII & 0.17 & 0.15 & 1.12 & 0.26 & & + \\
\hline & winRB & 0.21 & 0.14 & 1.49 & 0.14 & & \\
\hline \multirow{4}{*}{ 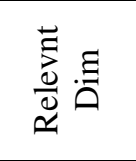 } & Rel & -7.28 & 0.30 & -24.57 & $<0.0001$ & $* * *$ & $* * *$ \\
\hline & $\times \mathrm{RB}$ & -0.83 & 0.59 & -1.42 & 0.16 & & \\
\hline & $\times$ wII & -1.81 & 0.85 & -2.13 & 0.033 & $*$ & $* *$ \\
\hline & $\times w R B$ & 2.70 & 0.81 & 3.34 & 0.0009 & $* * *$ & * \\
\hline \multirow{8}{*}{ 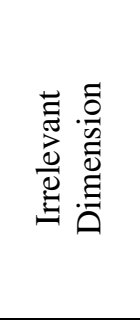 } & Irrel & -1.23 & 0.35 & -3.48 & 0.0005 & $* * *$ & $* * *$ \\
\hline & $\times \mathrm{RB}$ & -0.32 & 0.71 & -0.46 & 0.65 & & \\
\hline & $\times$ wII & 1.44 & 1.03 & 1.39 & 0.16 & & \\
\hline & $\times$ wRB & -0.72 & 0.97 & -0.74 & 0.46 & & \\
\hline & Irrel $^{2}$ & -0.08 & 0.31 & -0.27 & 0.79 & & \\
\hline & $\times \mathrm{RB}$ & 1.70 & 0.62 & 2.76 & 0.006 & $* *$ & * \\
\hline & $\times$ wII & -1.94 & 0.92 & -2.12 & 0.034 & $*$ & * \\
\hline & $x w R B$ & 0.79 & 0.83 & 0.96 & 0.34 & & \\
\hline \multirow{8}{*}{ 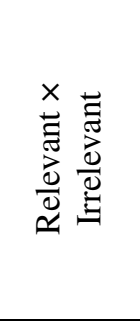 } & Rel $\times$ Irrel & -0.33 & 0.46 & -0.72 & 0.47 & & \\
\hline & $\times \mathrm{RB}$ & 3.05 & 0.92 & 3.32 & 0.0009 & $* * *$ & $* * *$ \\
\hline & $\times$ wII & -3.97 & 1.31 & -3.03 & 0.002 & $* *$ & $* *$ \\
\hline & $\times$ wRB & 1.02 & 1.29 & 0.79 & 0.43 & & \\
\hline & Rel $\times$ Irrel $^{2}$ & -1.10 & 1.32 & -0.83 & 0.41 & & \\
\hline & $\times \mathrm{RB}$ & 6.53 & 2.62 & 2.50 & 0.013 & $*$ & * \\
\hline & $\times$ wII & -1.32 & 3.71 & -0.36 & 0.72 & & + \\
\hline & $\times$ wRB & 0.62 & 3.64 & 0.17 & 0.86 & & \\
\hline \multirow{4}{*}{ 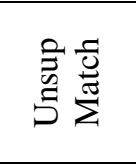 } & UnsupMatch & -0.16 & 0.10 & -1.58 & 0.11 & & \\
\hline & $\times \mathrm{RB}$ & -0.02 & 0.21 & -0.12 & 0.91 & & \\
\hline & $\times$ wII & -0.43 & 0.30 & -1.43 & 0.15 & & \\
\hline & $x w R B$ & -0.14 & 0.29 & -0.47 & 0.64 & & \\
\hline \multirow{4}{*}{ 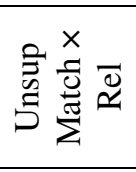 } & UnsupMatch $\times$ Rel & 0.08 & 0.59 & 0.14 & 0.89 & & \\
\hline & $\times \mathrm{RB}$ & 0.96 & 1.17 & 0.82 & 0.41 & & \\
\hline & $\times$ wII & -1.99 & 1.69 & -1.18 & 0.24 & & \\
\hline & $\times$ wRB & -0.42 & 1.62 & -0.26 & 0.80 & & \\
\hline \multirow{8}{*}{ 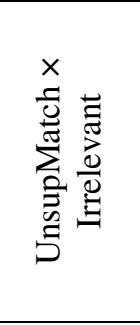 } & UnsupMatch $\times$ Irrel & 0.87 & 0.71 & 1.23 & 0.22 & & \\
\hline & $\times \mathrm{RB}$ & 1.05 & 1.41 & 0.75 & 0.45 & & \\
\hline & $\times$ wII & -1.43 & 2.03 & -0.71 & 0.48 & & \\
\hline & $\times w R B$ & -0.57 & 1.95 & -0.29 & 0.77 & & \\
\hline & UnsupMatch $\times$ Irrel $^{2}$ & -1.08 & 0.62 & -1.75 & 0.080 & + & \\
\hline & $\times \mathrm{RB}$ & 1.30 & 1.23 & 1.05 & 0.29 & & \\
\hline & $\times$ wII & 0.96 & 1.81 & 0.53 & 0.60 & & \\
\hline & $\times$ wRB & 0.21 & 1.67 & 0.13 & 0.90 & & \\
\hline \multirow{8}{*}{ 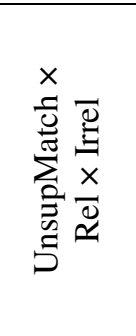 } & UnsupMatch $\times$ Rel $\times$ Irrel & -1.56 & 0.92 & -1.70 & 0.090 & + & \\
\hline & $\times \mathrm{RB}$ & -1.56 & 1.89 & -0.83 & 0.41 & & \\
\hline & $\times$ wII & 0.64 & 2.62 & 0.25 & 0.81 & & \\
\hline & $\times w R B$ & -2.13 & 2.73 & -0.78 & 0.43 & & \\
\hline & UnsupMatch $\times$ Rel $\times$ Irrel $^{2}$ & 2.61 & 2.60 & 1.00 & 0.32 & & \\
\hline & $\times \mathrm{RB}$ & -8.74 & 4.93 & -1.77 & 0.077 & + & $*$ \\
\hline & $\times$ wII & -3.98 & 6.33 & -0.63 & 0.53 & & \\
\hline & $x$ wRB & 12.09 & 7.97 & 1.52 & 0.13 & & * \\
\hline
\end{tabular}

$+\mathrm{p}<.1 ; * \mathrm{p}<.05, * * \mathrm{p}<.01, * * * \mathrm{p}<.001$ 


\section{S4. Supplementary Figures}
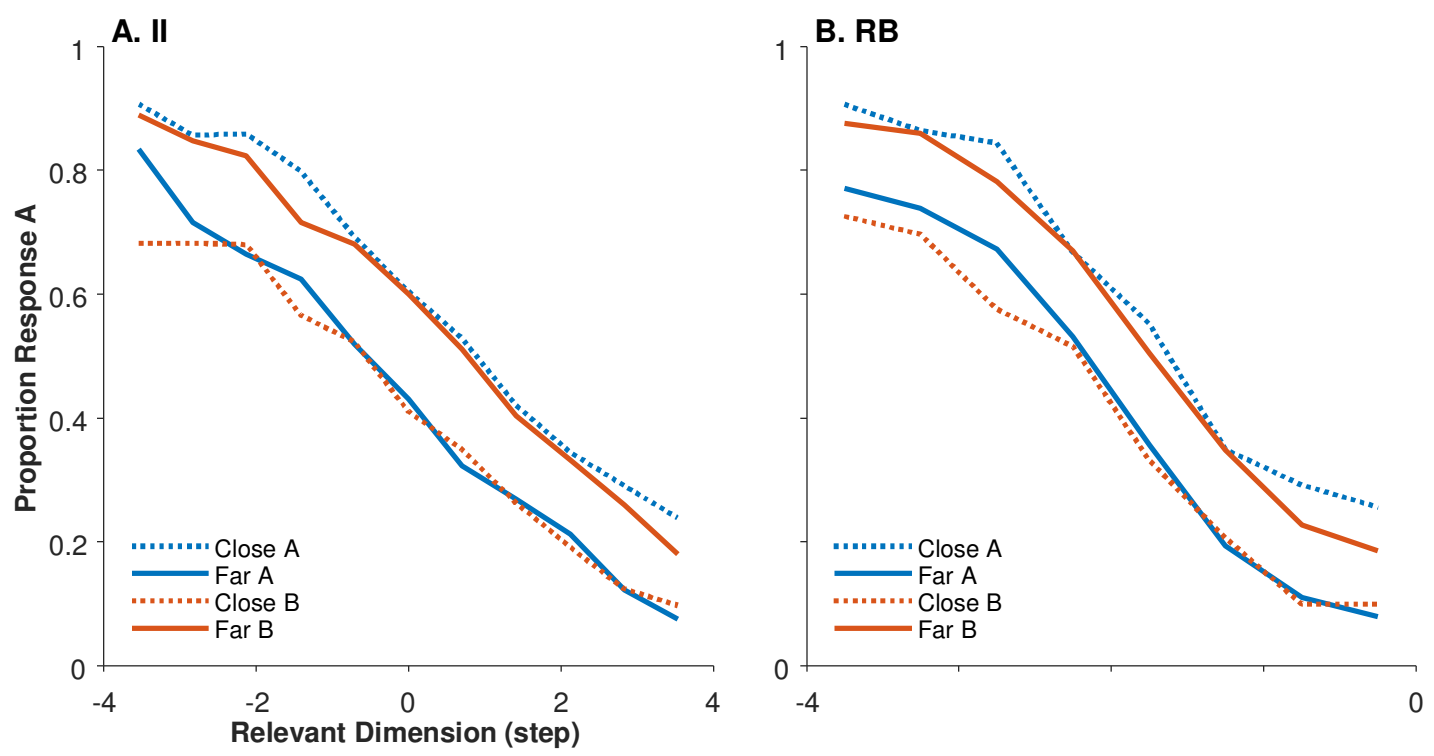

Figure S4.1: Effect of prior response (A vs. B) and the acoustic distance between the current and prior stimulus (based on a median split) on category judgements A) for the two II categories; B) for the two RB categories. When the stimulus was close to the prior one, and the participant responded category A (blue dashed curve) they were more likely to respond A again (an elevated curve); similarly if the stimulus was close to the current one, and they had said B before (red dashed curve), they were more likely to say B again. 

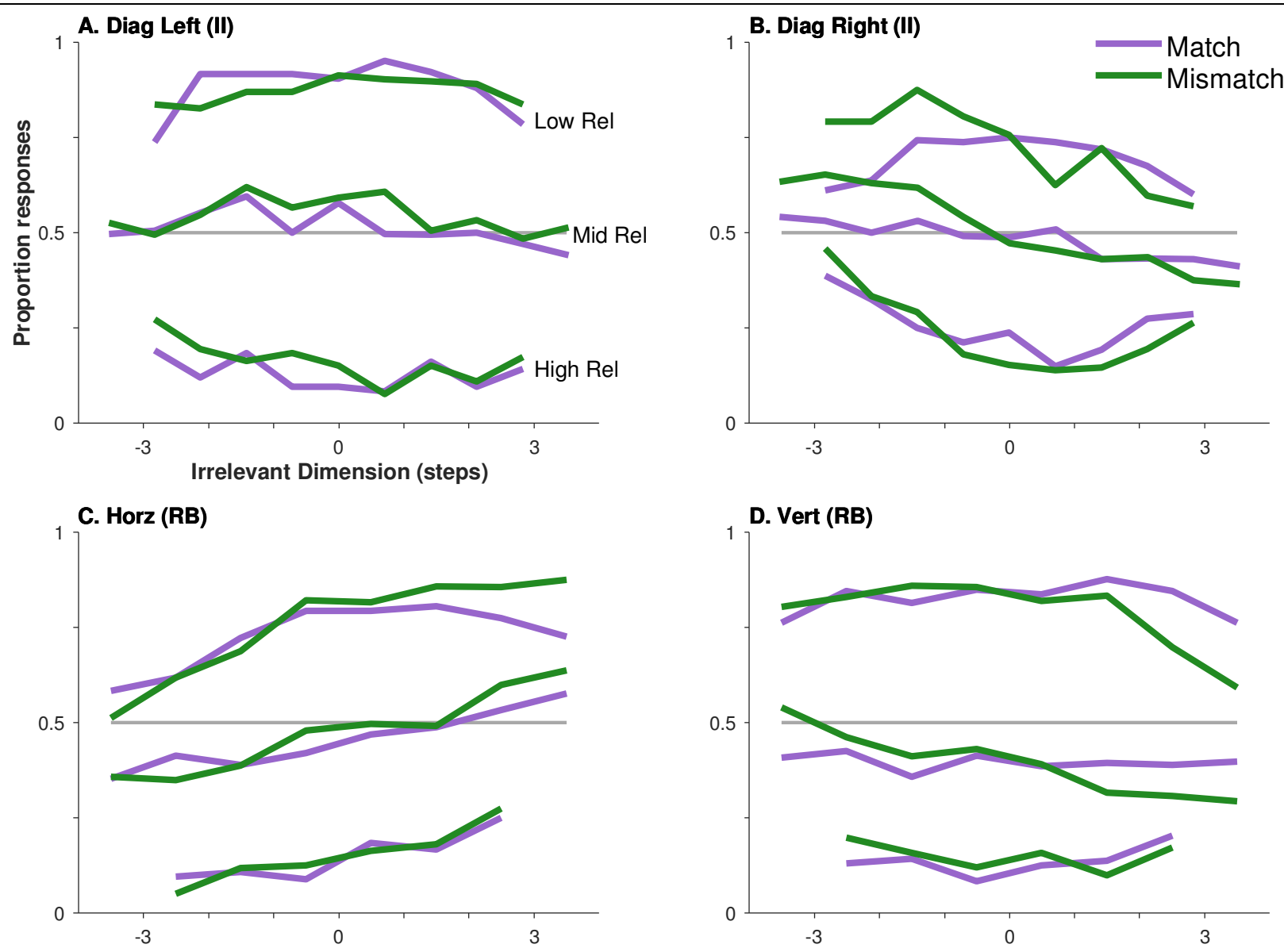

Figure S4.2: Effect of irrelevant dimension, supervised match and relevant dimension on Category A responses for all subjects. Relevant dimension is grouped by tertile (low, mid, high). Note that for diagonal-left, horizontal and vertical categories, an interaction of supervised match and the irrelevant dimension can be seen at low and mid values of the relevant dimension. For these curves, the purple curves (match) tended to be flatter (reflecting less utilization of irrelevant dimensions), while the green curves (mismatch) tended to show a systematic relationship between the irrelevant dimension and responses. 

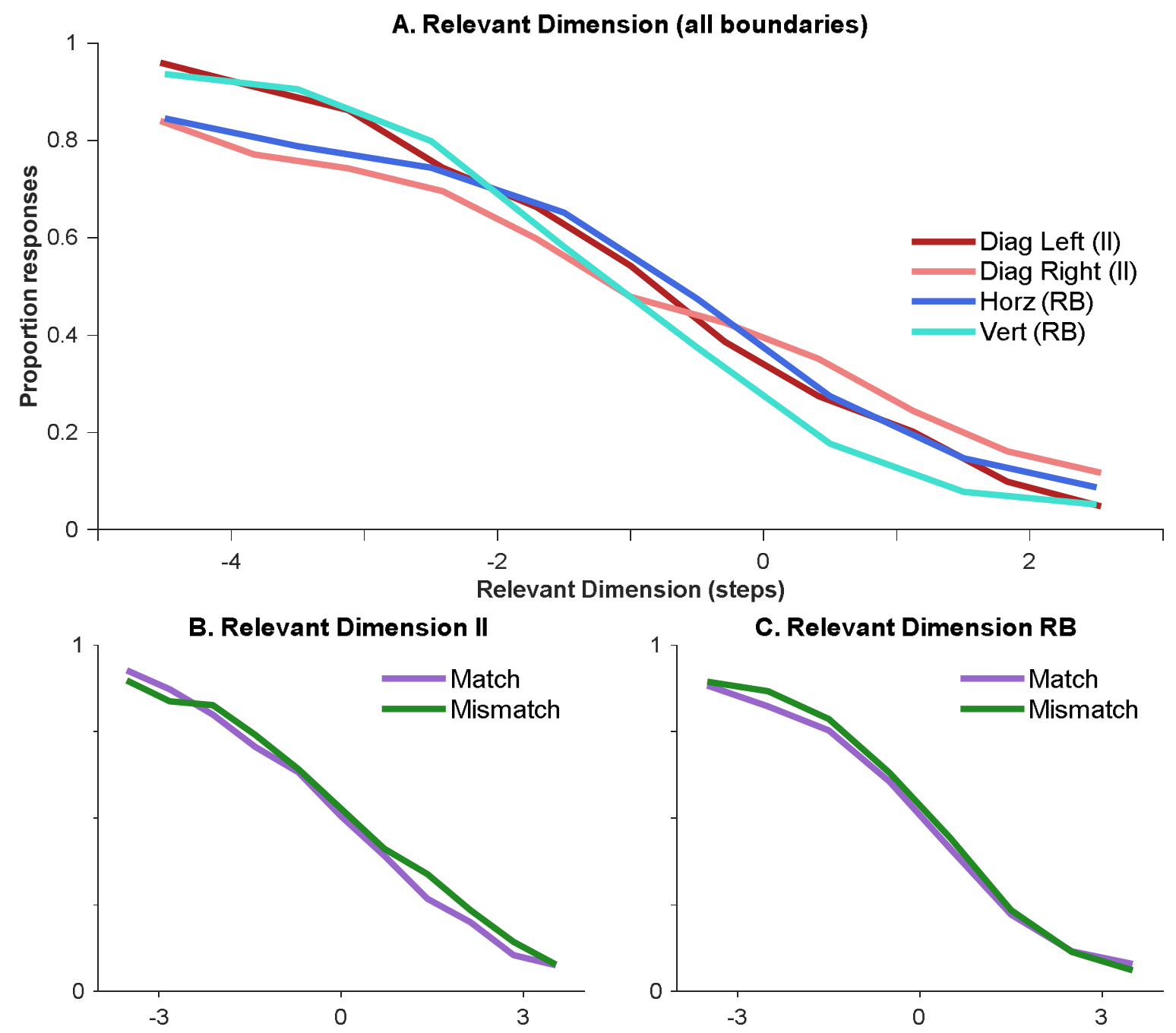

Figure S4.3: Effect of relevant dimension on categorization for the restricted set of subjects (compare to Figure 7 in main text). A) Effect of boundary type (averaging across the supervised / unsupervised match condition). B) Effect of supervised/unsupervised matched for the II boundaries. C) Effect of supervised/unsupervised matched for the RB boundaries. 

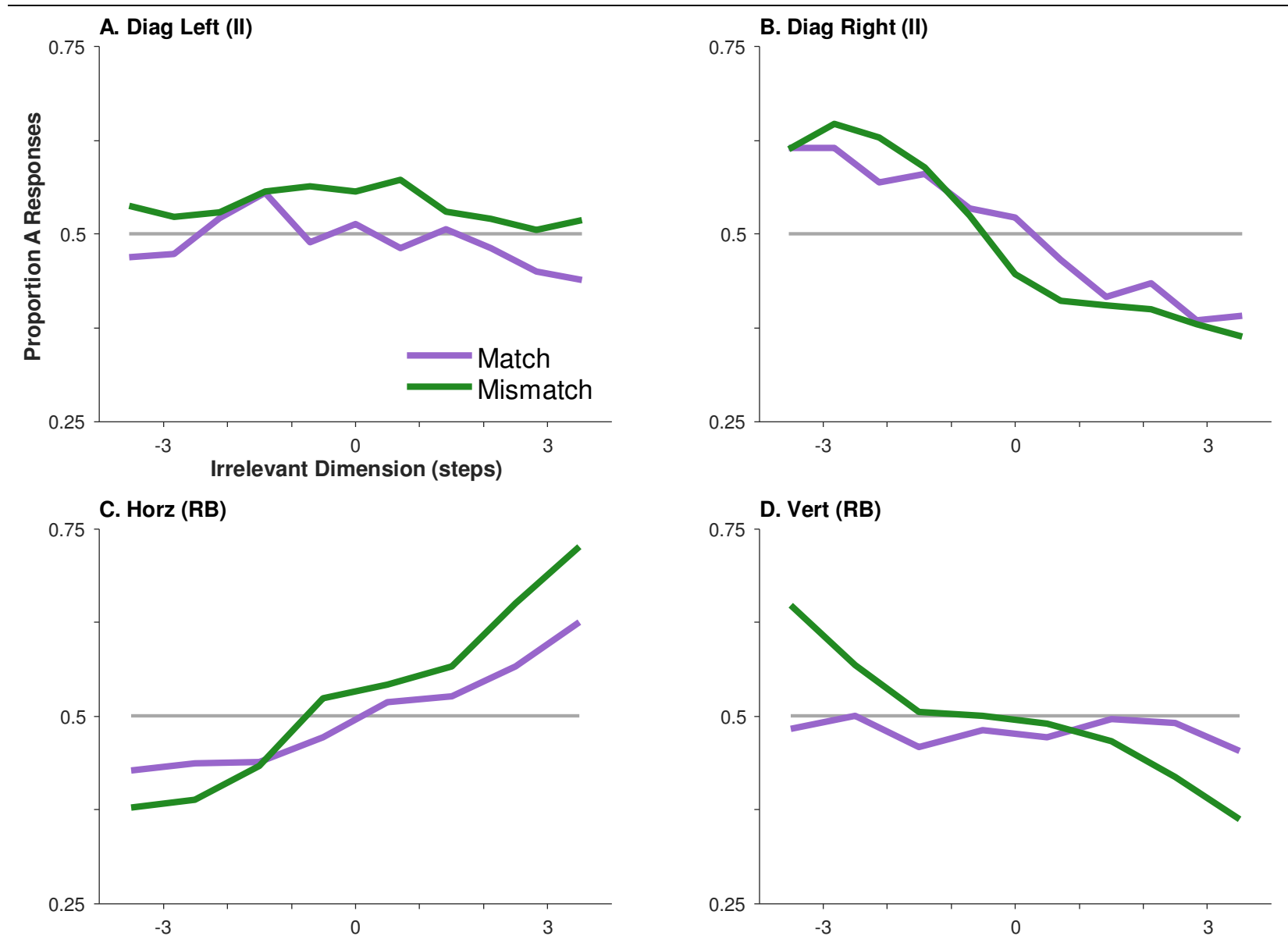

Figure S4.4: Effect of irrelevant dimension and supervised/unsupervised match on category A responses. If listeners are learning to ignore the irrelevant dimension, they should show a flat response at $50 \%$ (shown in gray lines). This is observed in both supervised/unsupervised match conditions in the diagonal-left condition (A). In the other three conditions (B-C), we see flatter functions when the supervised and unsupervised distributions match (in purple) than mismatch (green).

\section{References}

Ashby, F. G., \& Maddox, W. T. (2011). Human category learning 2.0. Annals of the New York Academy of Sciences, 1224(1), 147-161.

Chandrasekaran, B., Yi, H.-G., \& Maddox, W. T. (2014). Dual-learning systems during speech category learning. Psychonomic Bulletin \& Review, 21(2), 488-495. doi:10.3758/s13423-013-0501-5

Maddox, W. T., Chandrasekaran, B., Smayda, K., \& Yi, H.-G. (2013). Dual systems of speech category learning across the lifespan. Psychology and aging, 28(4), 1042-1056. doi:10.1037/a0034969

Roark, C. L., \& Holt, L. L. (2018). Task and distribution sampling affect auditory category learning. Attention, Perception, \& Psychophysics, 80(7), 1804-1822. doi:10.3758/s13414-018-1552-5

Yi, H.-G., \& Chandrasekaran, B. (2016). Auditory categories with separable decision boundaries are learned faster with full feedback than with minimal feedback. The Journal of the Acoustical Society of America, 140(2), 1332-1335. doi:10.1121/1.4961163 